\title{
JUCYS GRAPHS OF ANGULAR MOMENTUM THEORY
}

\author{
G. Merkelis \\ Vilnius University Research Institute of Theoretical Physics and Astronomy, A. Goštauto 12, LT-01108 Vilnius, Lithuania \\ E-mail: merkelis@itpa.lt
}

Received 14 May 2004

Dedicated to the 100th anniversary of Professor A. Jucys

\begin{abstract}
A brief review of the results obtained in Vilnius while developing the graphical methods of quantum angular momentum theory is presented. Numerous modifications and extensions of the original method proposed by A. Jucys, J. Levinson, and V. Vanagas are discussed. The application of the graphical method to the study of one-, two-electron as well as the effective operators and their matrix elements in the coordinate and second quantization representation is surveyed.
\end{abstract}

Keywords: atomic spectroscopy, graphical methods, angular momentum

PACS: $31.15 . \mathrm{Md}, 31.15 .-\mathrm{p}, 35.15 . \mathrm{Ar}$

\section{Introduction}

This paper is dedicated to mark the centenary of Prof. A. Jucys as well as to give a brief review of the results obtained in Vilnius while developing graphical methods of angular momentum theory. The majority of the basic results was obtained by Prof. A. Jucys with co-workers or under his close supervision. More recent investigations of the graphical method developed in the second quantization representation take a considerable part of the present paper.

More than forty years have passed since the basic elements and theorems of the graphical methods of the quantum angular momentum theory were established. Despite this long period of time in the contemporary papers dealing with theoretical spectroscopy one can frequently find the references to the book [1] where the foundations of the graphical methods were presented. This can be explained by the fact that the graphical methods of the angular momentum theory are still very useful for solving atomic, molecular, and nuclear spectroscopic problems. From the very beginning the use of the graphical approach allows us to avoid tedious and lengthy algebraic manipulations while deriving the analytical expressions and calculations by hand for relatively simple from the point of view of the presentday traditional problems: e. g., for the calculation of the matrix elements of the Hamiltonian or operator of the multipole radiation for atomic systems with one or several open shells. Nowadays, the scope of the problems where the graphical methods are used does not change considerably, however, the complexity of the tasks to be solved increases drastically. This is mainly due to the use of wide expansions of wave functions of the system under consideration in the computations. Then the order of the matrix for solving the eigenvalue problems reaches thousands and the calculation of matrix elements must be extremely fast. Here the graphical approach can help to derive the optimal expressions for the problem considered.

The graphical method of [1] was developed mainly on the basis of the results of [2], group theory studies of $[3,4]$, and tensor techniques of [5-10]. An important suggestion of [11] to consider the wave function of the angular momentum operator and irreducible tensor operators as the representatives of the irreducible tensorial sets opens the new possibilities in developing the algebraic and the graphical methods. A. Jucys and co-workers intensively improved the tensor operator formalism for the complex cases of atoms with open shells in the coordinate representation. These investigations were summarized in [12]. A more recent list of the publications on this topic can be found in [13]. At the same time, an effective technique of the calculation of matrix elements in the second quantization representation was considered (see, for example, $[14,15])$. The comprehensive studies of this method in Vilnius were summarized in [16] and [17]. The innovations of algebraic methods in the studies of the atomic structure cal- 
culations stimulated the developing and modification of the original graphical method [1]. In the present paper we shall try to describe the innovations carried out by the researchers in Vilnius.

Since the middle of the fifties, the activities in Vilnius for developing the graphical approach of quantum angular momentum theory can be observed. In a series of papers [18-20] the diagrammatic representation of the Wigner coefficients (WC) and their sum of products were proposed while treating the problems of angular momentum theory. Here also the construction and reduction of the complex diagrams composed of the Wigner coefficients were investigated. The generation, classification, and symmetric properties of $3 n j$ coefficients as cornerstone diagrams entering into the expressions of the graphical approach were intensively considered by many authors [21-25]. The results of the above-mentioned investigations were generalized and presented in the final version in the book [1]. The application of the graphical approach of [1] was illustrated in [26] while deriving a general expression of the matrix element for the scalar two-particle operator in the case of the antisymmetric two-electron wave fuctions. In the same issue in which [26] appeared, in a series of papers [27-29], the graphical approach based on the Clebsch-Gordan coefficients (CGC) instead of the Wigner coefficients has been developed. This approach and the studies of many other problems of the angular momentum theory were presented in the monograph [30]. (The second edition appeared in 1977 [31].) The idea of [11] of the irreducible tensorial sets was widely explored in [30]. The graphical representation of tensorial operators introduced in [32], their matrix and submatrix elements (important for the graphical method results) were also included in this book. The application of the graphical method of the angular momentum in the study of the perturbation theory (PT) expansions for atoms started with the paper [33]. Here it was proved that the angular momentum theory diagrams involved in the consideration of the Coulomb interactions in the case of closed shells are topologically equivalent to the Goldstone's diagrams [34] used in the diagrammatic Many-Body Perturbation Theory (MBPT). Open-shell PT was considered in $[35,36]$. The PT expansion for the Green's function of open-shell electrons was investigated in [35] for the Coulomb interactions. In [36] the PT expansion of the matrix element of the atomic transition operator was studied. Here the graphical representation of matrix elements by means of block diagrams corresponding to operator and many-electron states were given. Angular-momentum part of a submatrix element was presented by a closed diagram composed of the Clebsch-Gordan coefficients. The detailed consideration of graphical evaluation of matrix elements for one- and two-particle operators in the case of antisymmetric many-electron states by involving generalized coefficients of fractional parentage (CFP) was given in [37]. This approach was based on the algebraic method of generalized coefficients of fractional parentage [38]. A general technique of the derivation of the expression of matrix elements for one- and two-particle operators in the graphical way for nonorthogonal orbitals was developed in $[39,40]$.

Comprehensive reviews of earlier studies (19501980) in the development of the graphical methods and techniques were presented in $[41,42]$. Here we will single out only the investigations necessary for deeper understanding of our approach developed in the second quantization representation. The main modification of the graphical method of [1] introduced in [43] consists of rejecting the arrows for the basic graph of the Wigner coefficient. The arrow is involved for the definition of the sum of products of the Wigner coefficients. The studies of [44] and [42] start from the definition of the graphs for bra- and ket-vectors corresponding to the wave function of quantum system. This gives more space for the graphical considerations of various quantities, e.g., scalar product, projection operators. In [45-48] the graphical method of angular momentum theory to form the expressions for the effective operators corresponding to PT expansion terms is used. Here the open-shell cases were studied. For our investigations particularly useful are the considerations of [14] where the second quantization approach jointly with diagrammatic MBPT is discussed. In the paper [41] a detailed and consistent method of the graphical construction of the expressions of $N$-electron matrix elements for one- and two-particle operators as well as for the effective operators is developed in the coordinate representation. Finally, the book [49] accumulates, probably, the most extensive information not only of the graphical techniques used but it also contains numerical tables, formula, etc. for basic quantities of the angular momentum theory.

The second quantization approach developed in [14-16] is an efficient method to study many-electron states and matrix elements of operators for atomic interactions. For many problems of the open-shell atoms this method gives more elegant and simpler solutions in comparison to considerations performed in the coordinate representation [16]. In [50] the possibilities to 
use the graphical methods of angular momentum theory jointly with the second quantization approach while taking into account the tensorial properties of creation and annihilation operators in the perturbation expansion of the effective operator were discussed. This graphical technique was developed in [51-57]. We have chosen the graphical technique of [31] as the basis of the studies. In [53] the extension of the graphical method [31] consists of the introduction of arrows in the use of the Clebsch-Gordan coefficients. The diagrams as well as the algebraic expressions for the second-order effective operator in a coupled tensorial form were presented in $[52,57]$ where the open-shell case for the Coulomb interaction was investigated. The basic ideas of the graphical evaluation of matrix elements between the antisymmetric many-particle states by using second quantization formalism were formulated in $[55,58]$ and developed in [59]. Here the general rules for the construction and reduction of the diagrams for operators and matrix elements are presented. The possibility to derive graphically the relationship between the submatrix elements of operator and its conjugate is discussed. In $[60,61]$ the graphical way to construct the effective operator not only for MBPT but also for some other problems of atomic spectroscopy was considered. The graphical technique in a coupled form was considered in [62-64]. In these papers, however, the main attention was given to the study of the transformations of irreducible tensorial products of creation and annihilation operators attached to the oneand two-particle operators.

Note that in [65] the schematic expressions for the matrix elements of one- and two-particle operators were presented in terms of the one-particle coefficients of fractional parentage and the diagrams for recoupling coefficients by using the graphical method of [44]. The graphical technique of [48] was explored in [66] to generate the recoupling coefficients which arise due to the change of the coupling schemes for the creation and annihilation operators in the effective operator.

The paper is structured as follows. In Section 2 a brief review of the basic elements, rules, and theorems involved for the construction, transformation, and reduction of the sums of products of the Clebsch-Gordan or the Wigner coefficients are presented. Various techniques of the graphical method are discussed. The graphical representation of the irreducible tensorial operators is given in Section 3. Here also the construction and evaluation of submatrix elements are studied. Section 4 indicates the ways of graphical representation of one- and two-particle operators as well as PT expansion terms. Section 5 describes the graphical techniques of the derivation of the expressions for $N$-particle operators. The CR and SQR representations are considered. Computer codes using the graphical methods are discussed.

The following abbreviations are used in the paper: $\mathrm{CR}$, coordinate representation; CFP, coefficient of fractional parentage; CGC, Clebsch-Gordan coefficient; GCGC, generalized CGC; RME, reduced matrix element; SBE, submatrix element; SR, summation rule; SQR, second quantization representation; WC, Wigner coefficient. The diagrams of the present paper are classified by means of the symbol ${ }^{X}(A, B, C, \ldots)_{Y}$, where $X+1$ points to the section number, and $Y$ indicates the ordering number of a diagram. The capital letters $A, B, C, \ldots$ differentiate the subjects of the diagrams attached. Graphical relations (equations) in the figures are itemized by (a), (b), (c) etc.

\section{The basic elements of graphical representation}

In this section we discuss the basic elements and statements which make a background of the graphical approach of the angular momentum theory and which are introduced in [18-20] and developed in the final version in [1]. Although the Wigner coefficient (WC) is the main quantity of the mentioned papers, we will follow the methods in [30] and [31] where the ClebschGordan coefficient (CGC) is the fundamental element of the graphical approach. This study will be useful to us.

To introduce the main element of the graphical consideration, let us study the unitary transformation between the basis functions (referred to as uncoupled basis) formed from the products of the eigenstates $\left|j_{i} m_{i}\right\rangle$ of the angular momentum operators $j_{i}^{2}$ and $j_{z_{i}}$ to the basis of coupled wave functions [31]:

$$
\left|j_{1} j_{2} j_{3} m_{3}\right\rangle=\sum_{m_{1} m_{2}}\left[\begin{array}{ccc}
j_{1} & j_{2} & j_{3} \\
m_{1} & m_{2} & m_{3}
\end{array}\right]\left|j_{1} m_{1}\right\rangle\left|j_{2} m_{2}\right\rangle .
$$

Here the first factor on the right of (1) denotes the CGC (or vector coupling coefficient) [2]. The function $\left|j_{1} j_{2} j_{3} m_{3}\right\rangle$ is an eigenstate of the operators $j_{1}^{2}, j_{2}^{2}, j_{3}^{2}$, and $j_{z_{3}}$, where $j_{3}=j_{1}+j_{2}$. The CGC is related to the WC by (see, for example, [1])

$$
\begin{aligned}
& {\left[\begin{array}{ccc}
j_{1} & j_{2} & j_{3} \\
m_{1} & m_{2} & m_{3}
\end{array}\right]} \\
& \quad=(-1)^{j_{1}-j_{2}+m_{3}}\left[j_{3}\right]^{1 / 2}\left(\begin{array}{ccc}
j_{1} & j_{2} & j_{3} \\
m_{1} & m_{2} & -m_{3}
\end{array}\right) .
\end{aligned}
$$


Namely the WC (the last factor on the right of Eq. (2)) is the quantity for which the graph ${ }^{1} A_{1}$ has been introduced in [18]. Each line of ${ }^{1} A_{1}$ was associated with $j_{i}, m_{i}$. The arrow pointed to the free end of a line describes $m_{i}$, whereas the arrow pointed to a node indicates $-m_{i}\left(\right.$ see $\left.^{1} A_{2}\right)$. The node of the graph is supplied with "+" or "-" (positive or negative sign) if the arguments of the WC are ordered in counter-clockwise or clockwise direction. The change of a sign involves the phase factor $(-1)^{j_{1}+j_{2}+j_{3}}$.

The authors of [30] advise to use CGC instead of WC in a graphical evaluation. They stressed the simplicity of the suggested method as an advantage comparing it with other approaches. In this approach, the graph ${ }^{1} A_{3}$ denotes the CGC of (1). The heavy (thick) line is associated with the resultant momentum $j_{3}$ of the vector sum of $j_{1}$ and $j_{2}$. A sign at the node for CGC obeys the same rule as for WC, however, the change of a sign at the node involves the phase factor $(-1)^{j_{1}+j_{2}-j_{3}}$. The method of [31] is developed in such a way that there is no need to use arrows at all. Below, we will indicate the problems where the use of the arrows is still very effective in the CGC approach.

The symbols ${ }^{1} A_{1}$ and ${ }^{1} A_{3}$ are not the unique graphs used to represent the WC and CGC. For instance, in [43] and [48] the WC is denoted by ${ }^{1} A_{4}$. Then, for example, in [48] CGC is presented by a graph ${ }^{1} A_{5}$. In these techniques, the arrows are explored in a slightly different way from that defined in [1]. This aspect will be discussed later.

When generalizing Eq. (1) in the case of $k$ eigenstates, we write [31]

$$
\begin{aligned}
& \left.\mid\left(j_{1}, j_{2}, j_{3}, \ldots, j_{k}\right)^{A} \text { ajm }\right\rangle \\
& \quad=\sum_{m_{1} m_{2} \ldots m_{k}}\left[\begin{array}{ccc}
j_{1} & j_{2} \cdots & j \\
m_{1} & m_{2} \cdots m
\end{array}\right]_{a}^{A} \\
& \quad \times\left|j_{1} m_{1}\right\rangle\left|j_{2} m_{2}\right\rangle\left|j_{3} m_{3}\right\rangle \ldots\left|j_{k} m_{k}\right\rangle,
\end{aligned}
$$

where the first factor on the right of Eq. (3) is a generalized Clebsh-Gordan coefficient (GCGC) [31]. Notice that for GCGC in (3) $m_{1}+m_{2}+\cdots+m_{k}=m$. In the case when the WC is used instead of CGC in (3), the quantity corresponding to GCGC is referred to as a generalized WC [1]. A GCGC is given by the following expression [31]:

$$
\begin{aligned}
& {\left[\begin{array}{cccc}
j_{1} & j_{2} & \cdots & j \\
m_{1} & m_{2} & \cdots & m
\end{array}\right]_{a}^{A}=\sum_{m_{a}}\left[\begin{array}{ccc}
j_{1} & j_{2} & j_{12} \\
m_{1} & m_{2} & m_{12}
\end{array}\right]} \\
& \times\left[\begin{array}{ccc}
j_{12} & j_{3} & \cdots \\
m_{12} & m_{3} & \cdots
\end{array}\right] \cdots\left[\begin{array}{lll}
\cdots & j_{k} & j \\
\cdots & m_{k} & m
\end{array}\right] .
\end{aligned}
$$

Here $A$ denotes a coupling scheme of the momenta $j_{1}, j_{2}, j_{12}, \ldots, j_{k}, j$, and $a=\left\{j_{12}, \ldots\right\}$ represents the sequence of intermediate momenta. In (4) summation is carried out over all projections $m_{a}=$ $\left\{m_{12}, \ldots\right\}$ of $a$. For example, for the specific case when $k=4$ and when the coupling scheme $A=$ $\left\{j_{1} j_{2}\left(j_{12}\right), j_{3} j_{4}\left(j_{34}\right), j\right\}$ is chosen,

$$
\begin{aligned}
& {\left[\begin{array}{ccccc}
j_{1} & j_{2} & j_{3} & j_{4} & j \\
m_{1} & m_{2} & m_{3} & m_{4} & m
\end{array}\right]_{j_{12}, j_{34}}^{A}} \\
& =\sum_{m_{12} m_{34}}\left[\begin{array}{ccc}
j_{1} & j_{2} & j_{12} \\
m_{1} & m_{2} & m_{12}
\end{array}\right]\left[\begin{array}{ccc}
j_{3} & j_{4} & j_{34} \\
m_{3} & m_{4} & m_{34}
\end{array}\right] \\
& \quad \times\left[\begin{array}{ccc}
j_{12} & j_{34} & j \\
m_{12} & m_{34} & m
\end{array}\right],
\end{aligned}
$$

where $a=\left\{j_{12}, j_{34}\right\}$ and $m_{a}=\left\{m_{12}, m_{34}\right\}$.

Formulated in [1] a fundamental statement (below referred as summation rule (SR)) for the construction of a composite diagram for the CGC claims that the summation over twice-repeated magnetic quantum number $m_{i}$ in CGC is performed by joining the corresponding lines with $j_{i}, m_{i}$ [31]. By applying this rule to (5), we obtain the diagram ${ }^{1} B_{2}$ for GCGC formed from the product of three CGC (the diagrams ${ }^{1} B_{1},{ }^{1} B_{1}^{\prime}$, and ${ }^{1} B_{1}^{\prime \prime}$ ). Here a thin-thick line represents intermediate momenta $j_{12}$ and $j_{34}$. Usually, the block diagram ${ }^{1} B_{3}$ is associated with the GCGC (4) if a coupling scheme $A$ is not specified or is immaterial. We have to keep in mind that by working with the WC, a typical sum over $m_{i}$ has, for example, in the case of two WC (the diagrams ${ }^{1} B_{4}$ and $\left.{ }^{1} B_{4}^{\prime}\right)$, the following schematic form $[1,18]$ :

$$
\begin{aligned}
{ }^{1} B_{5} & =\sum_{m_{1}}(-1)^{j_{1}-m_{1}}{ }^{1} B_{4}{ }^{1} B_{4}^{\prime} \\
& =\sum_{m_{1}}(-1)^{j_{1}-m_{1}}\left(\begin{array}{ccc}
j_{1} & j_{2} & j_{3} \\
m_{1} & m_{2} & m_{3}
\end{array}\right)\left(\begin{array}{ccc}
j_{1} & j_{2}^{\prime} & j_{3}^{\prime} \\
-m_{1} & m_{2}^{\prime} & m_{3}^{\prime}
\end{array}\right) .
\end{aligned}
$$

Then the summation over $m_{i}$ is performed by connecting the lines of the graphs of the WC corresponding to 


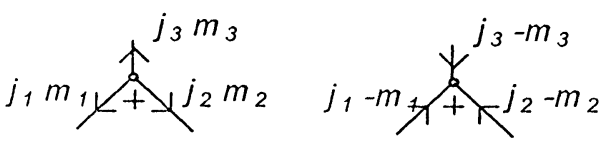

${ }^{1} A_{1}$

${ }^{1} A_{2}$<smiles>C[13CH]C([13CH3])[14CH3]</smiles>

${ }^{1} A_{3}$<smiles>CC(C)C</smiles>

${ }^{1} A_{4}$<smiles>CC(C)C(C)C(C)C</smiles>

${ }^{1} A_{5}$

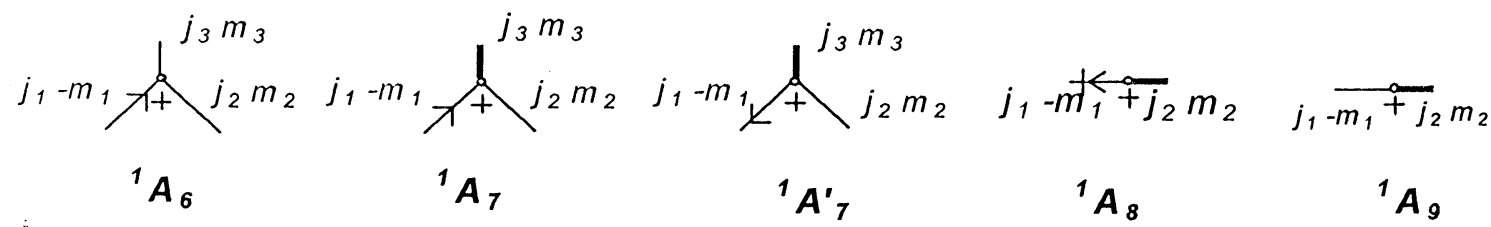

a)

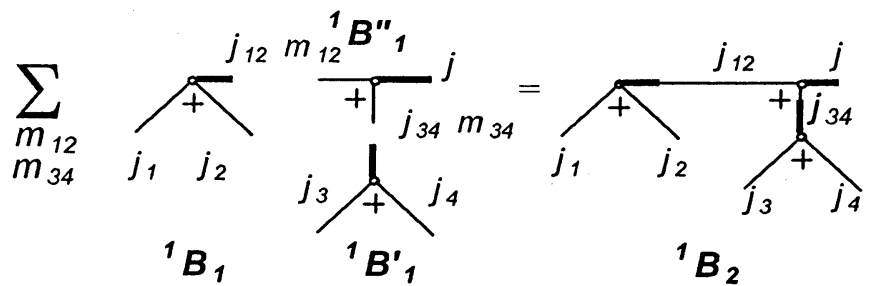

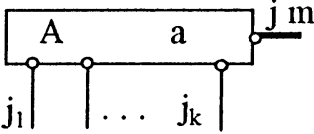

${ }^{1} B_{3}$

b)

c)

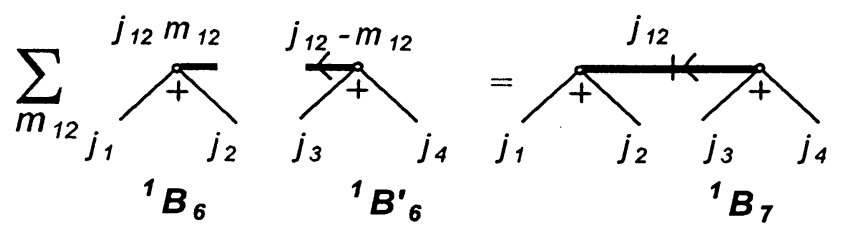

Fig. 1. The graph for the Clebsch-Gordan and Wigner coefficients and the sum of their products.

$\left(j_{i} m_{i}\right)$ and $\left(j_{i}-m_{i}\right)$ and by removing one arrow from the obtained line. Note that in [43] and [48] the graph ${ }^{1} A_{4}$ without arrows is used to denote WC. In their approach the line with the ingoing [48] (or outgoing [43]) arrow represents the $\mathrm{WC}$ with a negative parameter $-m$ which (WC) is multiplied by the phase factor $(-1)^{j+m}$. For example, the diagram ${ }^{1} A_{6}$ stands for

$$
(-1)^{j_{1}+m_{1}}\left(\begin{array}{ccc}
j_{1} & j_{2} & j_{3} \\
-m_{1} & m_{2} & m_{3}
\end{array}\right)
$$

In this case the summation over $m_{i}$ is carried out by connecting the lines corresponding to $m_{i}$ and $-m_{i}$.

The same convention of the arrow usage as explored in [48] for WC, in [53] was applied to CGC in order to present by a simple graphs the irreducible tensorial products of rank equal to zero (more details are given in Section 2). For this purpose, the diagram ${ }^{1} A_{7}$ was introduced to represent the CGC with $-m_{1}$ and multiplied by the phase factor $(-1)^{j_{1}+m_{1}}$. Furthermore, the outgoing arrow denotes the CGC with $-m_{1}$ but multiplied by $(-1)^{j_{1}-m_{1}}\left({ }^{1} A_{7}^{\prime}\right)$. Then, for example, the 


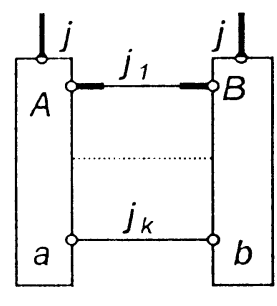

${ }^{1} c_{1}$

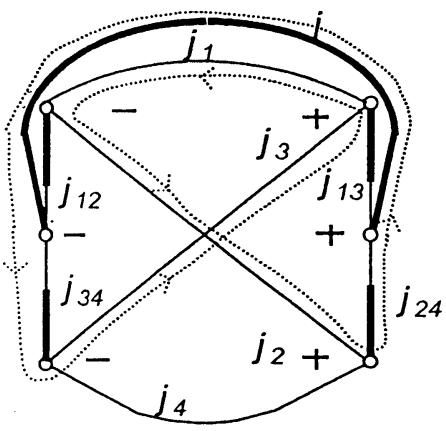

${ }^{1} c_{3}$

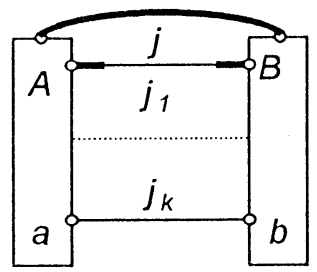

${ }^{1} c_{2}$

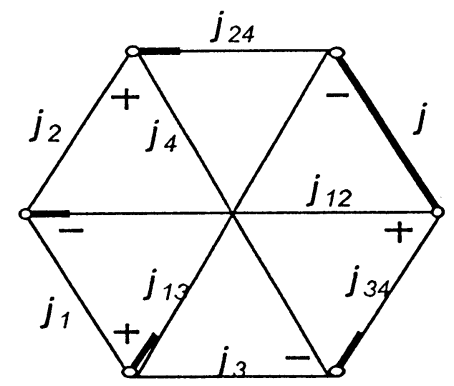

${ }^{1} C_{4}$

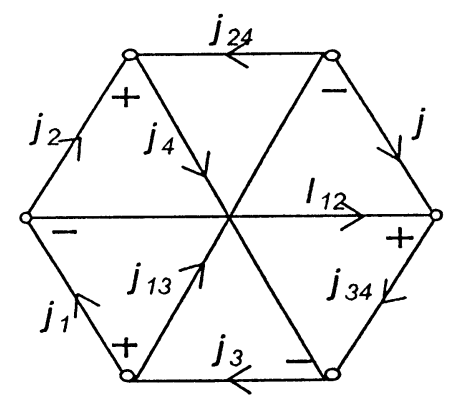

${ }^{1} C_{5}$

Fig. 2. Diagrams for recoupling coefficients and $3 n j$ coefficients.

GCGC (5) when $j=m=0$ is given by (see (c) of Fig. 1)

$$
\begin{aligned}
{ }^{1} B_{7}= & \sum_{m_{12}}{ }^{1} B_{6}{ }^{1} B_{6}^{\prime} \\
= & \sum_{m_{12}}\left[j_{12}\right]\left\{(-1)^{j_{12}-m_{12}}\left[j_{12}\right]^{-1 / 2}\left[\begin{array}{ccc}
j_{1} & j_{2} & j_{12} \\
m_{1} & m_{2} & m_{12}
\end{array}\right]\right. \\
& \left.\times\left[\begin{array}{ccc}
j_{3} & j_{4} & j_{12} \\
m_{3} & m_{4}-m_{12}
\end{array}\right]\right\} .
\end{aligned}
$$

In [53] the hyphen on the thick (thin) momentum line $j$ indicates that the diagram contains the factor $[j]^{1 / 2}\left([j]^{-1 / 2}\right)$. The factor $\left[j_{12}\right]$ in (7) was included following the convention of [31] about the graphical representation of diagrams with thick lines. Using the conventions of [53], the CGC (1) with resulting momentum $j_{3}=0$ is presented by the graph ${ }^{1} A_{8}$, whereas in the approach of [31] one has to use the same symbol ${ }^{1} A_{3}$ as for $j_{3} \neq 0$ indicating that $j_{3}=0$. The graph ${ }^{1} A_{8}$ as well as the convention (7) and the diagram ${ }^{1} A_{9}=\delta\left(j_{1} m_{1}, j_{3} m_{3}\right)$ (it represents the CGC when $j_{2}=0$ ) will be extensively used in our further considerations.
Let us return now to the approach of [31] and discuss the problem of transformation of the basis functions $\left|\left(j_{1}, j_{2}, j_{3}, \ldots, j_{k}\right)^{A} a j m\right\rangle$ with the coupling scheme $A$ to other basis functions $\left|\left(j_{1}, j_{2}, j_{3}, \ldots, j_{k}\right)^{B} b j m\right\rangle$ with the coupling $B$. The coefficients

$$
\begin{aligned}
R_{a b}= & \left\langle\left( j_{1}, j_{2}, j_{3}, \ldots, j_{k}^{A} a j m \mid\right.\right. \\
& \left.\times \mid\left(j_{1}, j_{2}, j_{3}, \ldots, j_{k}\right)^{B} \text { bjm }\right\rangle
\end{aligned}
$$

of such transformation are easily found by applying Eq. (3), the orthonormality condition $\left\langle j_{k} m_{k} \mid j_{k}^{\prime} m_{k}^{\prime}\right\rangle=$ $\delta\left(j_{k} m_{k}, j_{k}^{\prime} m_{k}^{\prime}\right)$, the diagram ${ }^{1} B_{3}$ and the SR. The summation of (3) leads to the diagram ${ }^{1} C_{1}$ which represents $R_{a b}$. Because the coefficient $R_{a b}$ is independent of $m$ (see [1] or [2]), it can be shown by the closed diagram ${ }^{1} C_{2}$ (a diagram which has no free lines) according to [1] and [31]. In [1] and [31] the diagram ${ }^{1} C_{2}$ is called a transformation matrix (TM) whereas in [43] it is referred to as a recoupling coefficient (RC) and $R_{a b}$ is denoted by

$$
\begin{aligned}
& {\left[\left(j_{1}, j_{2}, j_{3} \ldots j_{k}\right)^{A} a j \mid\left(j_{1}, j_{2}, j_{3}, \ldots, j_{k}\right)_{b}^{B} b j\right]} \\
& \equiv\left\langle\left(j_{1}, j_{2}, j_{3}, \ldots, j_{k}\right)^{A} a j m\right| \\
& \left.\quad \times \mid\left(j_{1}, j_{2}, j_{3}, \ldots, j_{k}\right)^{B} \text { bjm }\right\rangle
\end{aligned}
$$


For example, for the specific case $k=4$ and for the coupling schema $A=\left\{j_{1} j_{2}\left(j_{12}\right), j_{3} j_{4}\left(j_{34}\right), j\right\}$ and $B=\left\{j_{1} j_{3}\left(j_{13}\right), j_{2} j_{4}\left(j_{24}\right), j\right\}$, we can find $R_{a b}$ in the form ${ }^{1} C_{3}$. In order to give more convenient form (the form of a polygon) for the diagram ${ }^{1} C_{3}$ one can use the Hamilton line [1] to redraw ${ }^{1} C_{3}$. The Hamilton line of the diagram passes through each node once and after the Hamilton line is drawn, the diagram may be pulled apart so that this line lies along the edges of a polygon. In our case the Hamilton line is given by a dot line in ${ }^{1} C_{3}$. The redrawing of ${ }^{1} C_{3}$ gives the equivalent diagram ${ }^{1} C_{4}$. The comparison of ${ }^{1} C_{4}$ with the standard diagram used in [31] for the RC of four angular momenta yields

$$
\begin{gathered}
{\left[j_{1} j_{2}\left(j_{12}\right), j_{3} j_{4}\left(j_{34}\right), j \mid j_{1} j_{3}\left(j_{13}\right), j_{2} j_{4}\left(j_{24}\right), j\right]} \\
=\left[j_{12}, j_{34}, j_{13}, j_{24}\right]^{1 / 2}\left\{\begin{array}{lll}
j_{1} & j_{2} & j_{12} \\
j_{3} & j_{4} & j_{34} \\
j_{13} & j_{24} & j
\end{array}\right\} .
\end{gathered}
$$

The second factor in Eq. (9) is $9 j$ coefficient ( $9 j$ symbol) and represents a symmetric part of Eq. (9) (see, for example, [1]). The $9 j$ coefficient is a specific case of a $3 n j$ coefficient when $n=3(n+1$ is the number of angular momenta involved in the recoupling). In general, $3 n j$ coefficient is presented by the closed diagram formed from the WC. For instance, $9 j$ coefficient in (9) is given by ${ }^{1} C_{5}$ [1]. The diagram of $3 n j$ coefficient has $2 n$ nodes and $3 n$ parameters (lines), and $2 n-2$ intermediate momenta. It cannot be decomposed into two separate parts by cutting through three lines, however, arbitrary $3 n j$ coefficient can be expressed by multiple sums of products of $6 j$ coefficients. The exclusive role of $3 n j$ coefficients can be explained by the fact that in graphical evaluations of the arbitrary diagram one is trying to express it through $3 n j$ coefficients which analytical formulas are known. In a number of papers (see [31] for review) the generation, classification, symmetric properties, and nonvanishing conditions of $3 n j$ coefficients up to $n=9$ are considered. Of course, the $6 j$ and $9 j$ coefficients are the most frequently used quantities in the calculations. The more detailed discussion on this topic is out of the scope of our paper. The GCGC and $3 n j$ coefficients are the cornerstone elements of the graphical approach of the angular momentum theory [31].

If we sum the generalized CGC or WC at least over one pair of $m$ then one obtains the diagram with a cycle, i.e. in this diagram we can distinguish a polygon formed from the nodes. These diagrams are considered as the ones which have a closed part of a diagram [31]. In the present paper the name "diagram" (or $j m$ coefficient [1]) frequently will be attributed to such type of the diagrams in order to stress the difference from generalized CGC or WC. In the graphical evaluations it is convenient to represent the diagram under consideration in the standard form. A diagram is in the standard form if after the cutting all thin lines it breaks into several GCGC's in the way that all free lines of the original diagram are attributed to one GCGC. To present an arbitrary diagram in the standard form one can use the rules of [31]. Three basic theorems were formulated in [1] which allow a transformation and reduction of arbitrary diagram. Here we present these theorems adopted in [31] for CGC approach.

Theorem 1 (Expansion of a diagram). If the diagram

$$
{ }^{1} D_{1} \equiv F_{b b^{\prime}}\left(l_{1}, \ldots, l_{z} \begin{array}{c}
j_{1}, \ldots, j_{n}, j \\
m_{1}, \ldots, m_{n}, m
\end{array}\right)
$$

(where $m=\sum_{i=1}^{n} m_{i}$ ) is presented in the standard form and breaks up into two GCGC by cutting the lines $l_{1}, \ldots, l_{z}$, then

$$
{ }^{1} D_{1}=\sum_{a}{ }^{1} D_{2}(a){ }^{1} D_{3}(a),
$$

where the diagram ${ }^{1} D_{2}(a)$ represents the recoupling coefficient $R_{b b^{\prime} a}\left(l_{1}, \ldots, l_{z}, j_{1}, \ldots, j_{n}, j\right)$, which is obtained by "closing" ${ }^{1} D_{1}$ with $\operatorname{GCGC}{ }^{1} D_{3}(a)$, i. e. the diagram ${ }^{1} D_{2}(a)$ is obtained by connecting the free lines of the original diagram ${ }^{1} D_{1}$ with the corresponding lines of ${ }^{1} D_{3}(a)$ (see (a) of Fig. 3).

The theorem presented below was formulated in [30] for slightly more general case than that given in [1].

Theorem 2 (Decomposition of a diagram). If the diagram

$$
{ }^{1} E_{1} \equiv G_{b b^{\prime}}\left(l_{1}, \ldots, l_{z} \begin{array}{c}
j_{1}, \ldots, j_{x}, j_{x+1}, \ldots, j_{y} \\
m_{1}, \ldots, m_{y}, m_{x+1}, \ldots, m_{y}
\end{array}\right)
$$

satisfies the requirements:

(a)

$$
m_{y}=\sum_{i=1}^{y-1} m_{i},
$$

(b) at least one part of ${ }^{1} E_{1}$ is in the standard form after cutting the lines $l_{1}, \ldots, l_{z}$, and

(c) at least one part of ${ }^{1} E_{1}$ has a closed part of the diagram after cutting the lines $l_{1}, \ldots, l_{z}$, 
a)

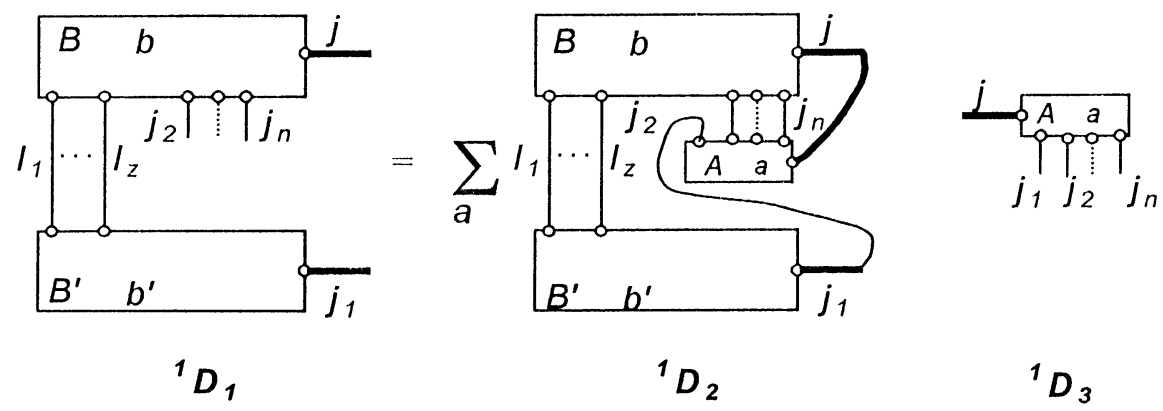

b)

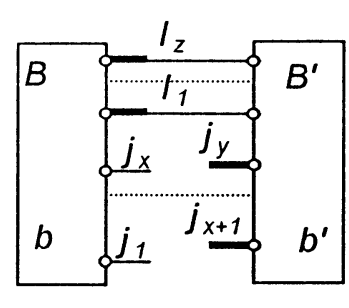

${ }^{1} E_{1}$

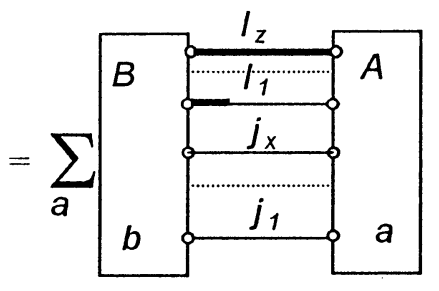

${ }^{1} E_{2}$

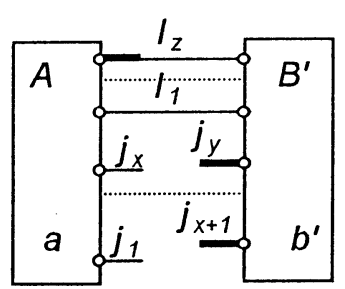

${ }^{1} E_{3}$

c)

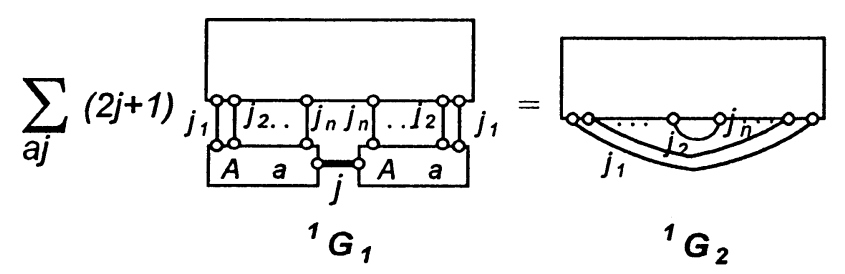

Fig. 3. Jucys, Levinson, and Vanagas Theorems.

then

$$
{ }^{1} E_{1}=\sum_{a}{ }^{1} E_{2}(a){ }^{1} E_{3}(a),
$$

where the diagram ${ }^{1} E_{2}(a)$ represents $R_{b a}\left(l_{1}, \ldots, l_{z}\right.$, $\left.j_{1}, \ldots, j_{x}\right)$ independent of $j_{x+1}, \ldots, j_{y}$, whereas $E_{a}$ corresponds to the diagram (see (b) of Fig. 3)

$$
\begin{aligned}
& { }^{1} E_{3}(a) \\
& \quad \equiv E_{a}\left(l_{1}, \ldots, l_{z} \begin{array}{l}
j_{1}, \ldots, j_{x}, j_{x+1}, \ldots, j_{y} \\
m_{1}, \ldots, m_{y}, m_{x+1}, \ldots, m_{y}
\end{array}\right) .
\end{aligned}
$$

Theorem 3 (Summation of diagrams). The summation of diagrams over momenta $a, j$ available in one or several diagrams is based on the property of GCGC
(GCGC is a matrix element of the unitary transformation [9]):

$$
\begin{aligned}
& \sum_{a j m}\left[\begin{array}{cccc}
j_{1} & j_{2} & \cdots & j \\
m_{1} & m_{2} & \cdots & m
\end{array}\right]_{a}^{A}\left[\begin{array}{cccc}
j_{1} & j_{2} & \cdots & j \\
m_{1}^{\prime} & m_{2}^{\prime} & \cdots & m
\end{array}\right]_{a}^{A} \\
& =\delta\left(m_{1}, m_{1}^{\prime}\right) \cdots \delta\left(m_{n}, m_{n}^{\prime}\right) .
\end{aligned}
$$

Here we shall demonstrate the application of (16) for the diagram ${ }^{1} G_{1}$ (see (c) of Fig. 3), i. e.

$$
{ }^{1} G_{2}=\sum_{a j}(2 j+1){ }^{1} G_{1}(a) .
$$

Note that Theorems 1 and 2 can be considered as special cases of the summation when one of the diagrams under summation is a recoupling coefficient. 


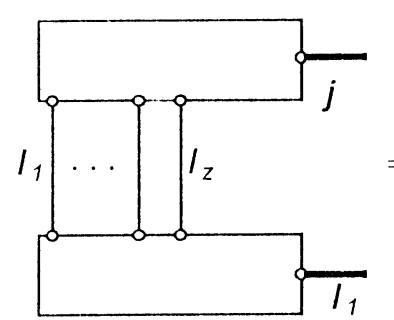

${ }^{1} D_{1}$

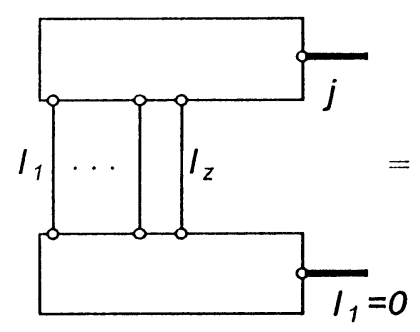

${ }^{1}{ }^{\prime \prime} 1$

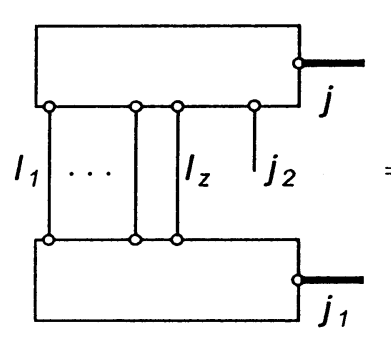

${ }^{1} D^{\prime \prime \prime} 1$

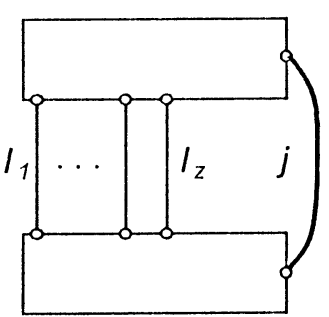

${ }^{1} D^{\prime}{ }_{2}$

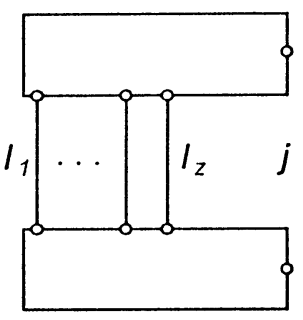

${ }^{1} D{ }_{2}$

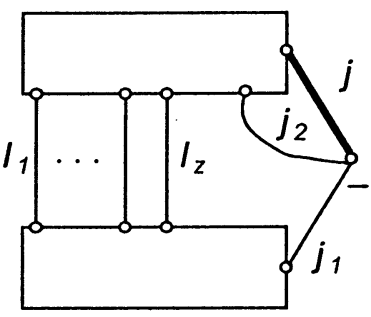

${ }^{1} D^{\prime \prime}{ }_{2}$
${ }_{j}{\widetilde{m_{1}}}_{j \mathrm{~m}}$

${ }^{1} D^{\prime}{ }_{3}$

$\underset{j m}{\circ}$

${ }^{1}{ }^{\prime \prime}{ }_{3}$
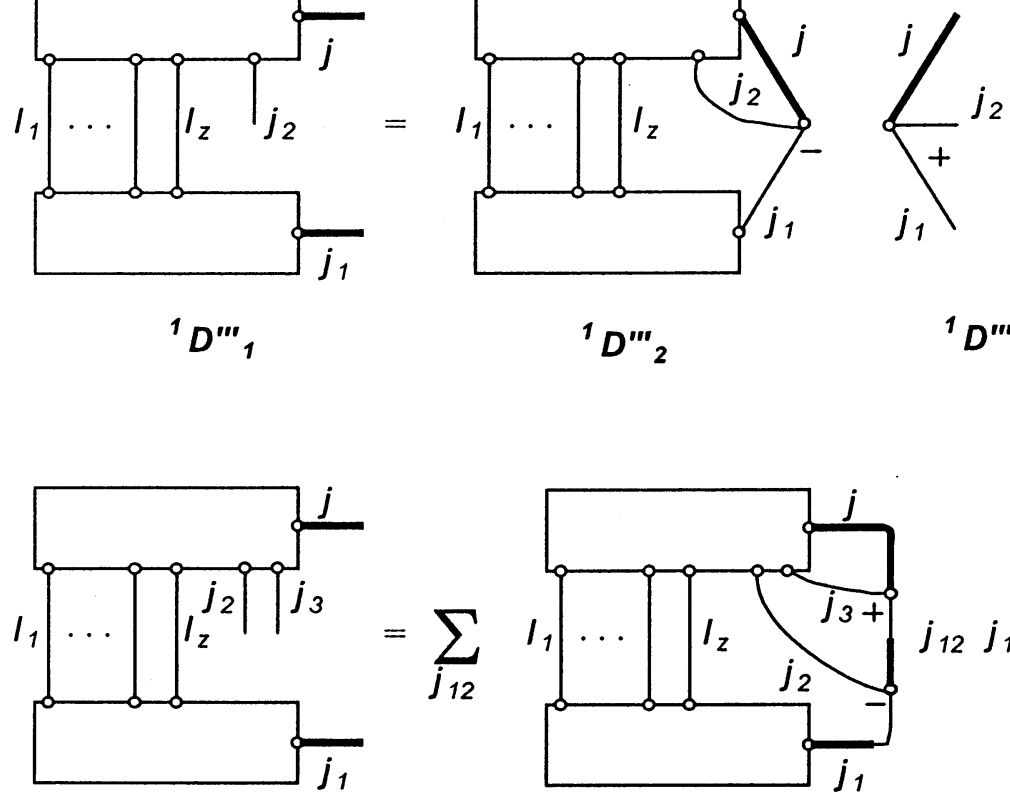

${ }^{1} D^{N}{ }_{1}$

${ }^{1} D^{N}{ }_{2}$

${ }^{1} D^{\prime \prime}{ }_{3}$

Fig. 4. Application of the Jucys, Levinson, and Vanagas Theorems (expansion of a diagram).

Consider some specific cases of Theorems 1-3. Suppose that in Eq. (10) $n=1$. Then we have the diagram with two free lines and immediately obtain that ${ }^{1} D_{1}^{\prime}={ }^{1} D_{2}^{\prime}{ }^{1} D_{3}^{\prime}$. Here ${ }^{1} D_{1}^{\prime}$ is a special case of ${ }^{1} D_{1}$ when $n=1$ and ${ }^{1} D_{3}^{\prime}=\delta\left(j_{1} m_{1}, j m\right)$. In addition, for example, when $j_{1}=0$, from ${ }^{1} D_{1}^{\prime}$ it follows that
${ }^{1} D_{1}^{\prime \prime}={ }^{1} D_{2}^{\prime \prime} D_{3}^{\prime \prime}$ (a diagram with one free line), where ${ }^{1} D_{3}^{\prime \prime}=\delta(j m, 00)[31]$ and ${ }^{1} D_{1}^{\prime \prime}$ is obtained from ${ }^{1} D_{1}^{\prime}$ by removing the line $j m$. In the case when ${ }^{1} D_{1}$ has only three free lines $(n=2)$, we have

$$
{ }^{1} D_{1}^{\prime \prime \prime}={ }^{1} D_{2}^{\prime \prime \prime}{ }^{1} D_{3}^{\prime \prime \prime}
$$


a) $z=1$
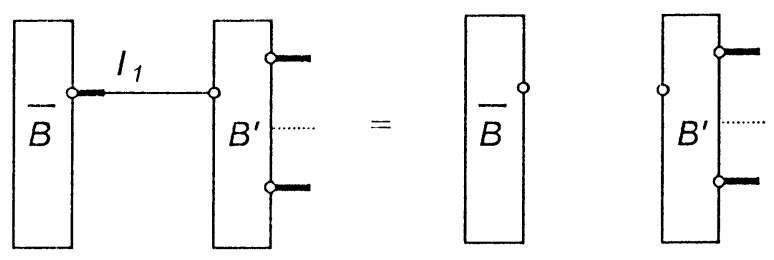

${\overline{I_{1}}}_{1}^{\circ}$

b) $z=2$

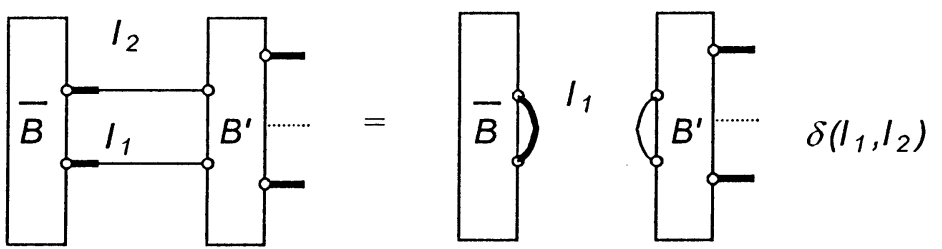

c) $z=3$

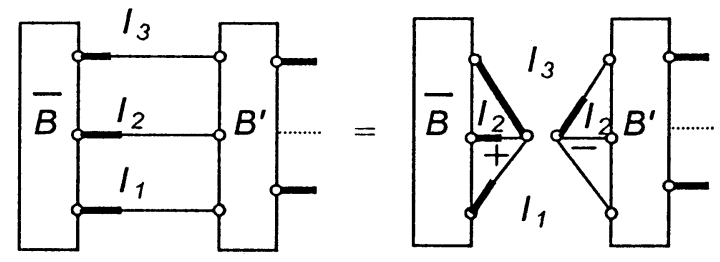

d) $z=4$

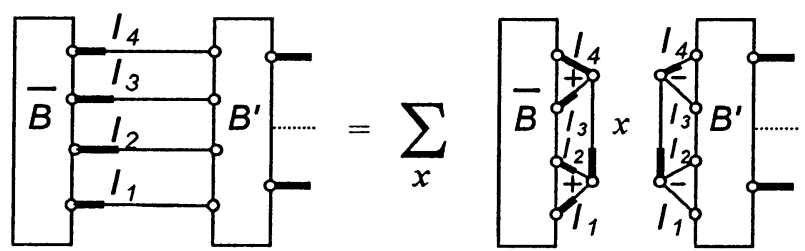

Fig. 5. Application of the Jucys, Levinson, and Vanagas Theorems (decomposition of a diagram).

The diagram ${ }^{1} D_{3}^{\prime \prime \prime}$ represents CGC while ${ }^{1} D_{2}^{\prime \prime \prime}$ is RC. Finally, suppose that $n=3$, then

$$
{ }^{1} D_{1}^{I V}=\sum_{j_{12}}{ }^{1} D_{2}^{I V}\left(j_{12}\right){ }^{1} D_{3}^{I V}\left(j_{12}\right) .
$$

Thus, when the diagram ${ }^{1} D_{1}$ has more than three free lines, then in contrast to previous cases, the sum over an intermediate momentum $j_{12}$ arises. Consider the special cases of Theorem 2. Suppose $x=0$, then the part of a diagram ${ }^{1} E_{1}$ with the coupling scheme $B$ is called a "closed" part and is usually denoted by $\bar{B}$. In Fig. 5 we present schematically the graphi- cal relations (a)-(d) corresponding to the cases when $z=1,2,3,4$.

Usually, the special cases of the Theorems 1-3 given in Figs. 4 and 5 are referred to as Jucys, Levinson, and Vanagas Theorems (JLV Theorems) (see, for example, $[43,46,48,81])$.

\section{The graphs for the irreducible tensors and submatrix elements}

In the previous section we studied the construction and reduction of the diagrams used for the recoupling tasks of eigenstates $|j m\rangle$. The $|j m\rangle$ itself has not been 
involved explicitly in the graphical evaluations of [1]. It has been done later in the paper [32]. There the graph ${ }^{2} A_{1}$ was introduced to denote the irreducible tensorial operator $T_{q}^{(k)}$ of rank $k$ with the projection $q$. The line of ${ }^{2} A_{1}$ was connected with $k, q$. Inside the semicircle of ${ }^{2} A_{1}$ an additional information about the operator $T_{q}^{(k)}$ was proposed for presentation. For example, this could be the information about the variables on which operator acts. In [32] it was also proposed an irreducible tensorial product

$$
\begin{aligned}
T_{q}^{(k)} & \equiv\left[T^{\left(k_{1}\right)} \times T^{\left(k_{2}\right)}\right]_{q}^{(k)} \\
& =\sum_{q_{1} q_{2}}\left[\begin{array}{lll}
k_{1} & k_{2} & k \\
q_{1} & q_{2} & q
\end{array}\right] T_{q_{1}}^{\left(k_{1}\right)} T_{q_{2}}^{\left(k_{2}\right)}
\end{aligned}
$$

of the irreducible tensors $T^{\left(k_{1}\right)}$ and $T^{\left(k_{2}\right)}$ associated with the graph ${ }^{2} A_{2}$. Obviously, this graph is obtained by using the graphs ${ }^{1} A_{3}$ and ${ }^{2} A_{1}$ assuming that the sums over $q_{1}$ and $q_{2}$ graphically are performed by joining the lines corresponding to the same $k_{i}, q_{i}$. Keeping in mind that the eigenstates $|j m\rangle$ as well as the operators $T_{q}^{(k)}$ form the irreducible tensorial set [11], the graph ${ }^{2} A_{1}$ can also be attributed to $|j m\rangle$. Then, the graphical procedures and rules given in Section 1 can be considered as a device for manipulation with the irreducible tensorial products.

The next contribution of [32] in the development of the graphical technique was related with the introducing of the graph for a submatrix element (SBE [31]) or reduced matrix element (RME [11]) of $T^{(k)}$ and with formulation of the diagrammatic way of obtaining the expression for SBE for complex products of irreducible tensorial operators. The Wigner-Eckart theorem (see, for example, $[11,16])$

$$
\begin{aligned}
& {\left[\alpha_{1} j_{1} m_{1}\left|T_{q}^{(k)}\right| \alpha_{2} j_{2} m_{2}\right]} \\
& \quad=(-1)^{\varphi}\left[\begin{array}{ccc}
j_{2} & k & j_{1} \\
m_{2} & q & m_{1}
\end{array}\right]\left[\alpha_{1} j_{1}\left\|T^{(k)}\right\| \alpha_{2} j_{2}\right]
\end{aligned}
$$

establishes the basis for this study. Let us emphasize that $\varphi=0$ is used in [31] and [32] whereas in SQR studies it is convenient to choose $\varphi=2 k[14,16])$. In [32] a matrix element on the left of Eq. (21) was proposed to associate with the graph ${ }^{2} A_{3}$ while the reduced element $\left[\alpha_{1} j_{1}\left\|T^{(k)}\right\| \alpha_{2} j_{2}\right]$ was expressed by symbol ${ }^{2} A_{4}$. Then the symbol ${ }^{2} A_{4}$ was obtained by applying the SR to ${ }^{2} A_{3}$ and the graph for the CGC in Eq. (21). Note that in the present paper we use the convention that $\left[\alpha_{1} j_{1}\|I\| \alpha_{2} j_{2}\right]=\delta\left(\alpha_{1} j_{1}, \alpha_{2} j_{2}\right)$ but $\left(\alpha_{1} j_{1}\|I\| \alpha_{2} j_{2}\right)=\delta\left(\alpha_{1} j_{1}, \alpha_{2} j_{2}\right) \sqrt{[j]}$, and $[j] \equiv 2 j+1$ (see, e. g., [31]). Here $I$ is the unit operator.

To understand the principles of graphical derivation of expressions for the RME for the complicated cases, consider the specific case

$$
\left[j_{1} j_{2} j\left\|\left[A^{\left(k_{1}\right)} \times B^{\left(k_{2}\right)}\right]^{(k)}\right\| j_{1}^{\prime} j_{2}^{\prime} j^{\prime}\right]
$$

when the operators $A^{\left(k_{1}\right)}$ and $B^{\left(k_{2}\right)}$ act on $\left|j_{1} m_{1}\right\rangle$ and $\left|j_{2} m_{2}\right\rangle$, respectively. The graphical derivation of expressions for the RME presented in [32] imitates the algebraic manipulation while obtaining this expression. Keeping in mind that

$$
\begin{aligned}
& {\left[j_{1} j_{2} j m\left|\left[A^{\left(k_{1}\right)} \times B^{\left(k_{2}\right)}\right]_{q}^{(k)}\right| j_{1}^{\prime} j_{2}^{\prime} j^{\prime} m^{\prime}\right]} \\
& =\sum_{m_{1}} \sum_{m_{2}}\left[\begin{array}{ccc}
j_{1}^{\prime} & j_{2} & j \\
m_{1} & m_{2} & m
\end{array}\right]\left[\begin{array}{ccc}
j_{1}^{\prime} & j_{2}^{\prime} & j^{\prime} \\
m_{1}^{\prime} & m_{2}^{\prime} & m^{\prime}
\end{array}\right] \\
& \quad \times\left[\begin{array}{cc}
k_{1} & k_{2} k \\
q_{1} & q_{2}
\end{array}\right]\left[j_{1} m_{1}\left|A_{q_{1}}^{\left(k_{1}\right)}\right| j_{1}^{\prime} m_{1}^{\prime}\right] \\
& \quad \times\left[j_{2} m_{2}\left|B_{q_{2}}^{\left(k_{2}\right)}\right| j_{2}^{\prime} m_{2}^{\prime}\right]
\end{aligned}
$$

and by using the symbols ${ }^{2} A_{3},{ }^{2} A_{4}$ and the WignerEckart theorem as well as by applying the SR, the diagram ${ }^{2} A_{5}$ is obtained. After separation of the graphs ${ }^{2} A_{4}\left(A^{\left(k_{1}\right)}\right)$ (it corresponds to $\left[\alpha_{1} j_{1}\left\|A^{\left(k_{1}\right)}\right\| \alpha_{1}^{\prime} j_{1}^{\prime}\right]$ ) and ${ }^{2} A_{4}\left(B^{\left(k_{2}\right)}\right)$ (it corresponds to $\left[\alpha_{2} j_{2}\left\|B^{\left(k_{2}\right)}\right\| \alpha_{2}^{\prime} j_{2}^{\prime}\right]$ ) from ${ }^{2} A_{5}$, the final expression for the SBE was found:

$$
\begin{aligned}
& {\left[j_{1} j_{2} j\left\|\left[A^{\left(k_{1}\right)} \times B^{\left(k_{2}\right)}\right]^{(k)}\right\| j_{1}^{\prime} j_{2}^{\prime} j^{\prime}\right]} \\
& \quad={ }^{2} A_{5} \\
& \quad={ }^{1} \bar{C}_{4}{ }^{2} A_{4}\left(A^{\left(k_{1}\right)}\right){ }^{2} A_{4}\left(B^{\left(k_{2}\right)}\right) .
\end{aligned}
$$

Here the diagram ${ }^{1} \bar{C}_{4}$ represents $\mathrm{RC}{ }^{1} C_{4}$, considered in Section 1. To find the analytical expression for ${ }^{1} \bar{C}_{4}$, one has to replace the parameters of ${ }^{1} C_{4}$ with the corresponding parameters of ${ }^{1} \bar{C}_{4}$, keeping in mind that the closed diagram does not change its value if all the signs at the nodes are replaced by the opposite ones [31]. Generalizing the considered procedure, one can claim that the SBE of arbitrary complexity can be given by the sum of products of $\mathrm{RC}$ and the submatrix elements of operators which make the original tensorial product. In $[30,31]$ the comprehensive rules for the graphical construction and evaluation of the expressions for reduced matrix elements are presented.

At this point we have to stress that a brief discussion connected with the fundamental rules and theorems of [31] (and, of course, [1]) used for the graphi- 
cal manipulation with irreducible tensors could be finished. The specific feature of this approach consists of avoiding the use of arrows in the diagrammatic manipulations. This, of course, greatly simplifies the procedures of derivation of expressions and the method considered is especially effective for the study of closed diagrams. However, the problems can occur when studying the irreducible tensorial products with the resulting rank equal to zero (we are forced to draw the line which moment $j$ is equal to zero) or when applying this method to the evaluation of $N$-electron operators or matrix elements in SQR. In Section 1 we have already discussed the modification of [31] approach throughout introducing the arrows in the graphical representation of CGC. Here we shall concentrate on the extensions applied to the graph ${ }^{2} A_{1}$ that enables one to represent graphically algebraic operations not considered in [31].

Note that in a number of methods $[42,44,49]$ the graphs for bra $\langle j m|$ and ket $|j m\rangle$ states, as well as the scalar product $\left\langle j m \mid j^{\prime} m^{\prime}\right\rangle$ and projection operator $P_{j}=$ $\sum_{m}|j m\rangle\langle j m|$ are the crucial elements of the graphical representation. For instance, in [44] the graphs ${ }^{2} B_{1}$ and ${ }^{2} B_{2}$ are associated with $|j m\rangle$ and $\langle j m|$, respectively. Furthermore, $|j-m\rangle$ and $\langle j-m|$ are given by ${ }^{2} B_{3}$ and ${ }^{2} B_{4}$. Then the graphical relation follows immediately (see (a) of Fig. 6):

$$
{ }^{2} B_{2}=(-1)^{j-m}{ }^{2} B_{3}
$$

of

$$
\langle j m|=| j m\rangle^{+}=(-1)^{j-m}|j-m\rangle,
$$

which defines the standard phase system [31]. However, in [42] differently from the approach of [44], $|j m\rangle$ and $\langle j m|$ are associated with ${ }^{2} B_{5}$ and ${ }^{2} B_{6}$. The scalar product $\left\langle j m \mid j^{\prime} m^{\prime}\right\rangle=\delta\left(j m, j^{\prime} m^{\prime}\right)$ in these methods is represented by the diagrams ${ }^{2} B_{7}$ and ${ }^{2} B_{8}$, respectively. The projection operator $P_{j}$ is given by ${ }^{2} B_{9}$ [44] and ${ }^{2} B_{10}$ [42].

In $[59,60]$ the extensions of the use of the tensors of [31] to account for the properties just considered were discussed. Here we have to emphasize that we explore the phase system of [14] and [16] when

$$
T_{q}^{(k)+}=(-1)^{k+q} \widetilde{T}_{-q}^{(k)},
$$

where $\widetilde{T}^{(k)}$ denotes the operator conjugated to $T^{(k)}$, and $T^{(k)+}$ is the tensor Hermitian conjugated to $T^{(k)}$. To single out the graphical notations for the operators $T_{q}^{(k)}$ and $\widetilde{T}_{q}^{(k)}$ in the CR, the graph ${ }^{2} C_{1}$ is associated with the operator $\widetilde{T}_{q}^{(k)}$. It was suggested that the diagrams ${ }^{2} C_{2}$ and ${ }^{2} C_{3}$ represent the expressions $(-1)^{k+q} A_{-q}^{(k)}$ and $(-1)^{k-q} A_{-q}^{(k)}$, respectively. Here $A_{q}^{(k)}$ can be either $T_{q}^{(k)}$ or $\widetilde{T}_{q}^{(k)}$. In addition, the action of the second arrow on the line $k$ (when already one arrow exists) is easily understood from the graphical relations (e) and (f) in Fig. 6. In [60] it was also assumed that the graphical relation $(\mathrm{g})\left({ }^{2} A_{1}\right)^{+}={ }^{2} C_{4}$ represents the operation of the Hermitian conjugation (26). The modifications introduced for the graph ${ }^{2} A_{1}$ of $T_{q}^{(k)}$ allows the additional manipulations with the symbol for irreducible tensor and are very useful in the construction of a graphical representation of the irreducible tensorial products and SBEs. For example, keeping in mind that an irreducible tensorial product of rank zero of two tensors, for instance, $\widetilde{A}^{(1)}$ and $B^{(1)}$, is given by the diagram [53]

$$
{ }^{2} C_{5}=\left[\widetilde{A}^{(1)} \times B^{(1)}\right]^{(0)}
$$

and by using the convention (g) of Fig. 6 when $\widetilde{A}=A$, the diagram ${ }^{2} C_{5}$ can be transformed into the graph ${ }^{2} C_{6}$ (here we suppose that the arrow is attributed to $\widetilde{A}$ ). Thus, ${ }^{2} C_{6}$ can be regarded as the graph which represents the scalar product $\left(A^{[1]} \cdot B^{(1)}\right)$ of two vectors, when $A^{[1]}$ defines the contrastandard component of $A$ [31].

A very useful concept for graphical representation of a reduced matrix element was introduced in [55] and developed in $[58,59]$ by using the relation [16]

$$
\begin{aligned}
& {\left[\alpha_{1} j_{1}\left\|T^{(k)}\right\| \alpha_{2} j_{2}\right]} \\
& =(-1)^{j_{2}-k-j_{1}}\left[j_{1}\right]^{-1 / 2} \\
& \quad \times\left[\left\langle\widetilde{\alpha_{1} j_{1}}\right| \times\left[T^{(k)} \times\left|\alpha_{2} j_{2}\right\rangle\right]^{\left(j_{1}\right)}\right]^{(0)}
\end{aligned}
$$

In Eq. (28) $\left[\left\langle\widetilde{\alpha_{1} j_{1}}\right| \times\left[T^{(k)} \times\left|\alpha_{2} j_{2}\right\rangle\right]^{\left(j_{1}\right)}\right]^{(0)}$ is the tensorial product with the resulting rank equal to zero. Then the right-hand side of Eq. (28) can be given by the diagram ${ }^{2} D_{1}$. The dashed line in ${ }^{2} D_{1}$ represents integration over the coordinates. This integration is completed when the semicircles associated with $\left\langle\widetilde{\alpha_{1} j_{1} m_{1}}\right|, T_{q}^{(k)}$, and $\left|\alpha_{2} j_{2} m_{2}\right\rangle$ are joined into a circle, and we obtain the diagram ${ }^{2} D_{2}$ which represents the SBE $\left[\alpha_{1} j_{1}\left\|T^{(k)}\right\| \alpha_{2} j_{2}\right]$. The diagrams ${ }^{2} D_{2}$ and ${ }^{2} A_{4}$ for the SBE $\left[\alpha_{1} j_{1}\left\|T^{(k)}\right\| \alpha_{2} j_{2}\right]$ account for the different phase factor used in [31] and [16] in the definition of the Wigner-Eckart theorem (21). In the case when $k=0,{ }^{2} D_{2}(k=0)={ }^{2} D_{3}=\delta\left(\alpha_{1} j_{1}, \alpha_{2} j_{2}\right)$.

Below we present the diagrams which will be used in our further consideration. The diagram ${ }^{2} D_{4}$ is asso- 


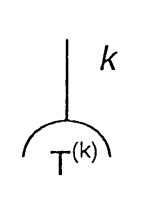

${ }^{2} A_{1}$

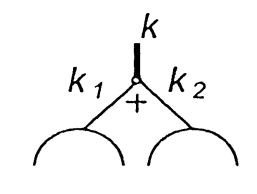

${ }^{2} \mathrm{~A}_{2}$

$k_{1}$

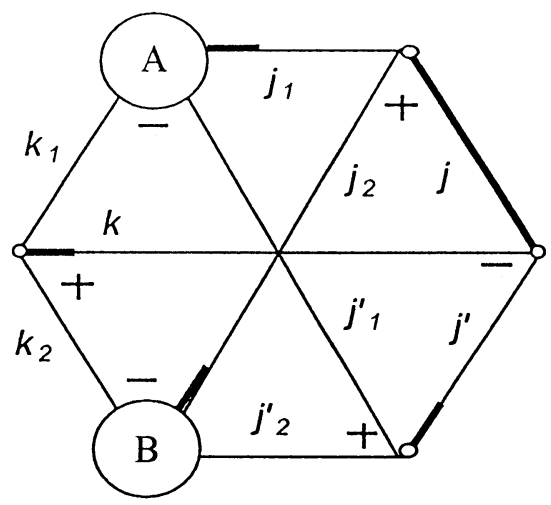

${ }^{2} A_{5}$
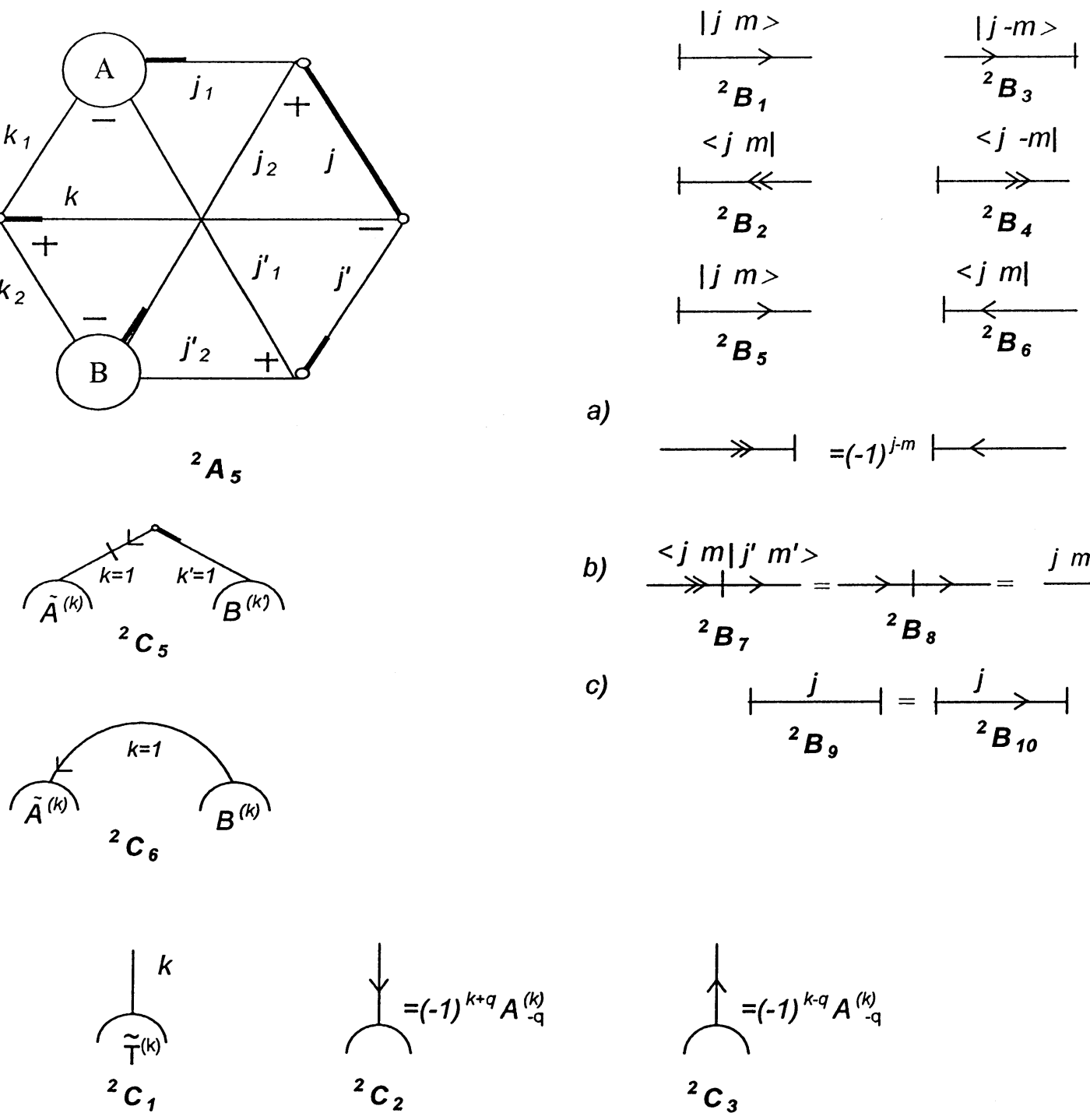

a)

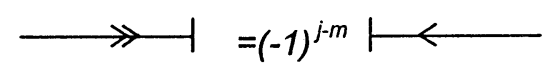

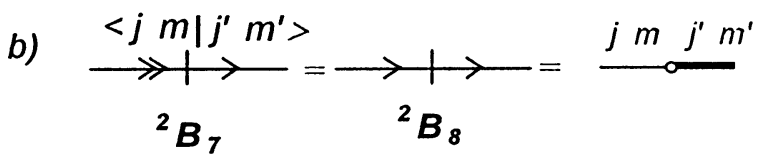

c)

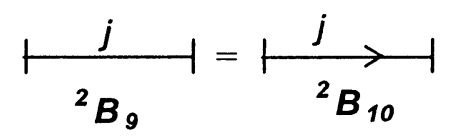

e) $\Psi=\$=(-1)^{2 k}$<smiles>CC(=CC(C)C)C(C)(C)C(C)(C)C(C)C(C)C</smiles><smiles>CC(C)=CC(C)=CC(C)C(C)C</smiles>

Fig. 6. The graph for irreducible tensors and submatrix elements I. 

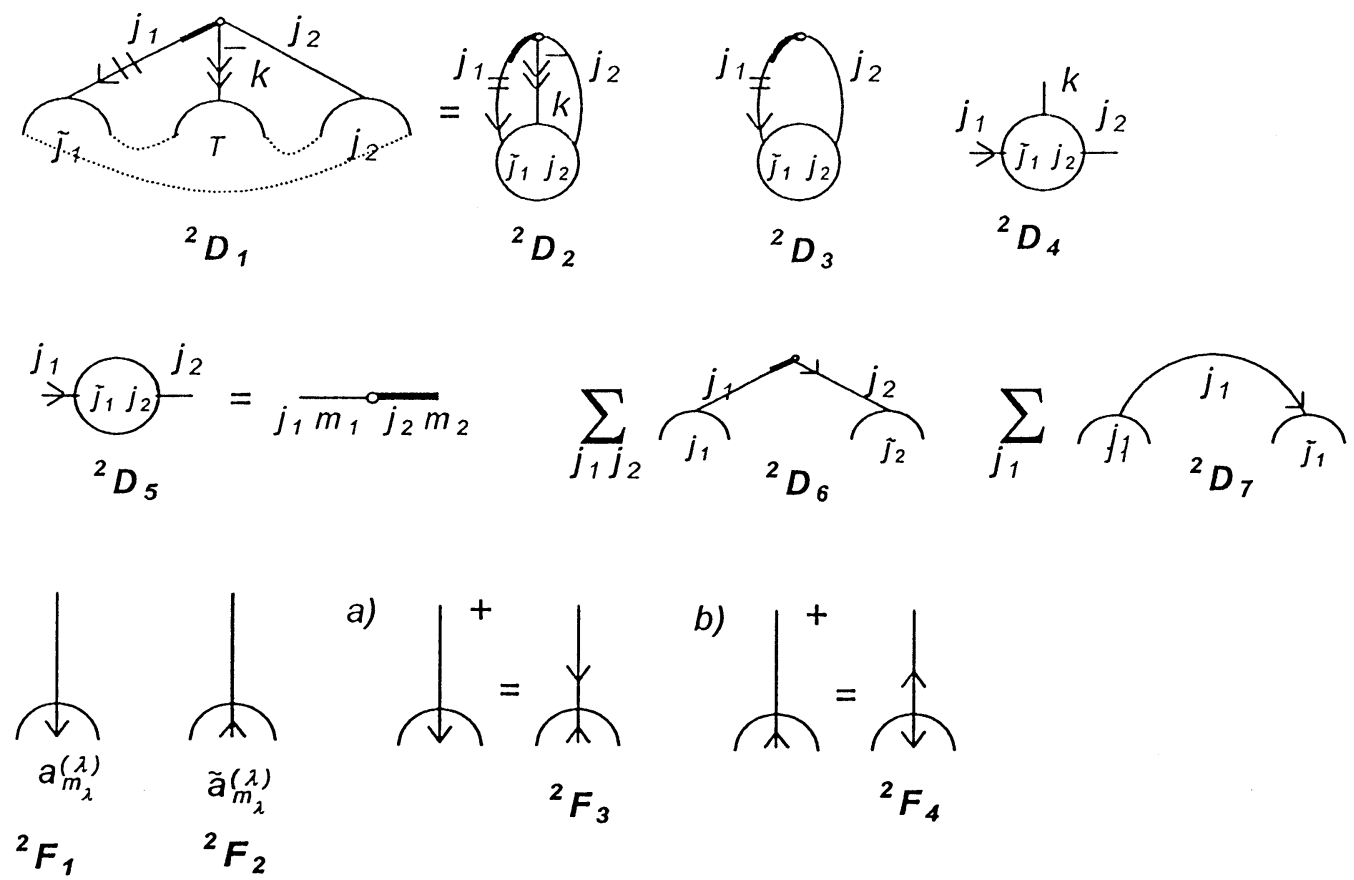

a)

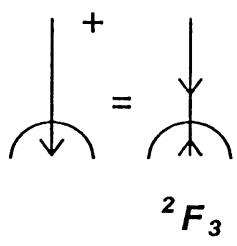

b)

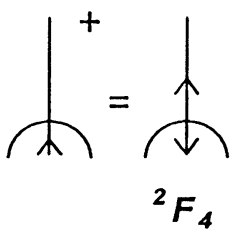

c)

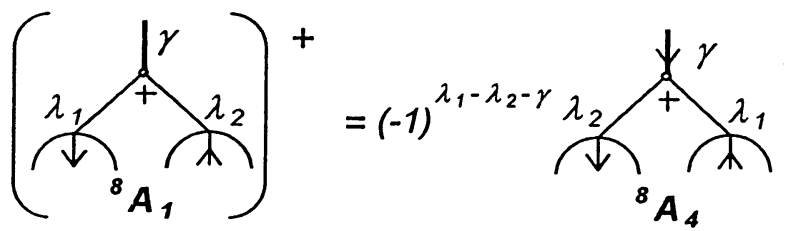

Fig. 7. The graph for irreducible tensors and submatrix elements II.

ciated with the matrix element $\left[\alpha_{1} j_{1} m_{1}\left|T_{q}^{(k)}\right| \alpha_{2} j_{2} m_{2}\right]$ and for the specific case $k=0$, we have ${ }^{2} D_{4}(k=0)=$ ${ }^{2} D_{5}=\delta\left(j_{1} m_{1}, j_{2} m_{2}\right)={ }^{1} A_{9}$. Finally, the projection operator $P_{j}$ can be given by the diagram ${ }^{2} D_{6}$ or ${ }^{2} D_{7}$. The diagram ${ }^{2} D_{7}$ is understood in the sense defined below (27).

Now we shall illustrate the use of the definition (28) in the considerations of complex SBE. For example, after the adaptation of the diagram ${ }^{2} D_{1}$ to $\left[j_{1} j_{2} j\left\|\left[A^{\left(k_{1}\right)} B^{\left(k_{2}\right)}\right]^{(k)}\right\| j_{1}^{\prime} j_{2}^{\prime} j^{\prime}\right],{ }^{2} E_{1}$ (see Fig. 8) represents the original diagram for the SBE in interest. The brackets \langle\rangle (it is an alternative notation of a dashed line in ${ }^{2} D_{1}$ ) indicate the integration over coordinates. The half-way diagram ${ }^{2} E_{2}$ is given to demonstrate which operators (they are put side-byside) should be coupled in order to form the diagrams for SBEs $\left[\alpha_{1} j_{1}\left\|A^{\left(k_{1}\right)}\right\| \alpha_{1}^{\prime} j_{1}^{\prime}\right]$ and $\left[\alpha_{2} j_{2}\left\|B^{\left(k_{2}\right)}\right\| \alpha_{2}^{\prime} j_{2}^{\prime}\right]$, respectively. By using the rules of Section 1, we decompose the diagram ${ }^{2} E_{2}$ in the following form:

$$
{ }^{2} E_{1}={ }^{2} E_{2}={ }^{2} E_{3}{ }^{2} E_{4}{ }^{2} E_{5} .
$$

This equation coincides with Eq. (23) because after the minor manipulations the diagram ${ }^{2} E_{3}$ transforms to ${ }^{1} \bar{C}_{4}$, while ${ }^{2} E_{4}$ and ${ }^{2} E_{5}$ correspond to ${ }^{2} A_{4}\left(A^{\left(k_{1}\right)}\right)$ and ${ }^{2} A_{4}\left(B^{\left(k_{2}\right)}\right)$, respectively. Here we have to point out some features which make the present approach different from that of [31]. First, in the present approach the diagram of a SBE is not a symbol, preventing any further transformations with it, but it is an element of a graphical technique with definite tensorial structure. Second, the derivation of the expression for complex SBE is considered as the task of recoupling the moments of an irreducible tensorial product. In the derivation a graph for a matrix element is not involved. This gives an advantage when treating complicated tensorial products.

In our diagrammatic studies of PT expansion of the effective operators $[50,53-55,58,59]$ the SQR for many-electron states and operators reveals itself as a very effective approach to study open-shell systems. Here we present some results of those studies related with the electron creation $a_{\lambda m_{\lambda}}$ and annihilation $a_{\lambda m_{\lambda}}^{+}$ operators considering them as irreducible tensorial op- 


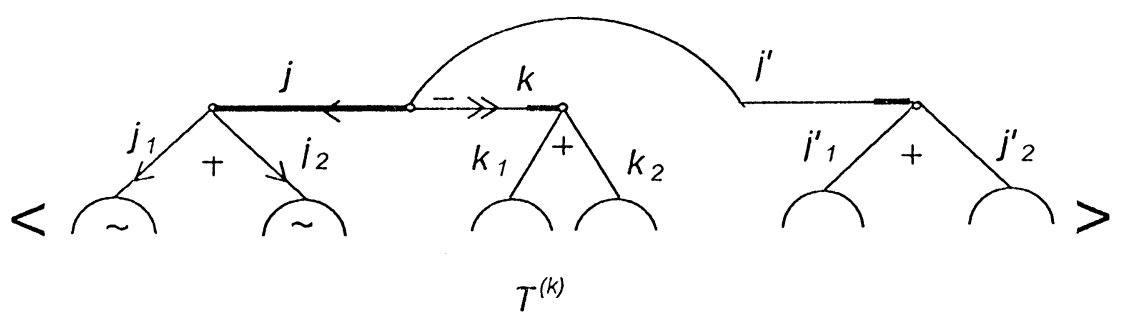

${ }^{2} E_{1}$
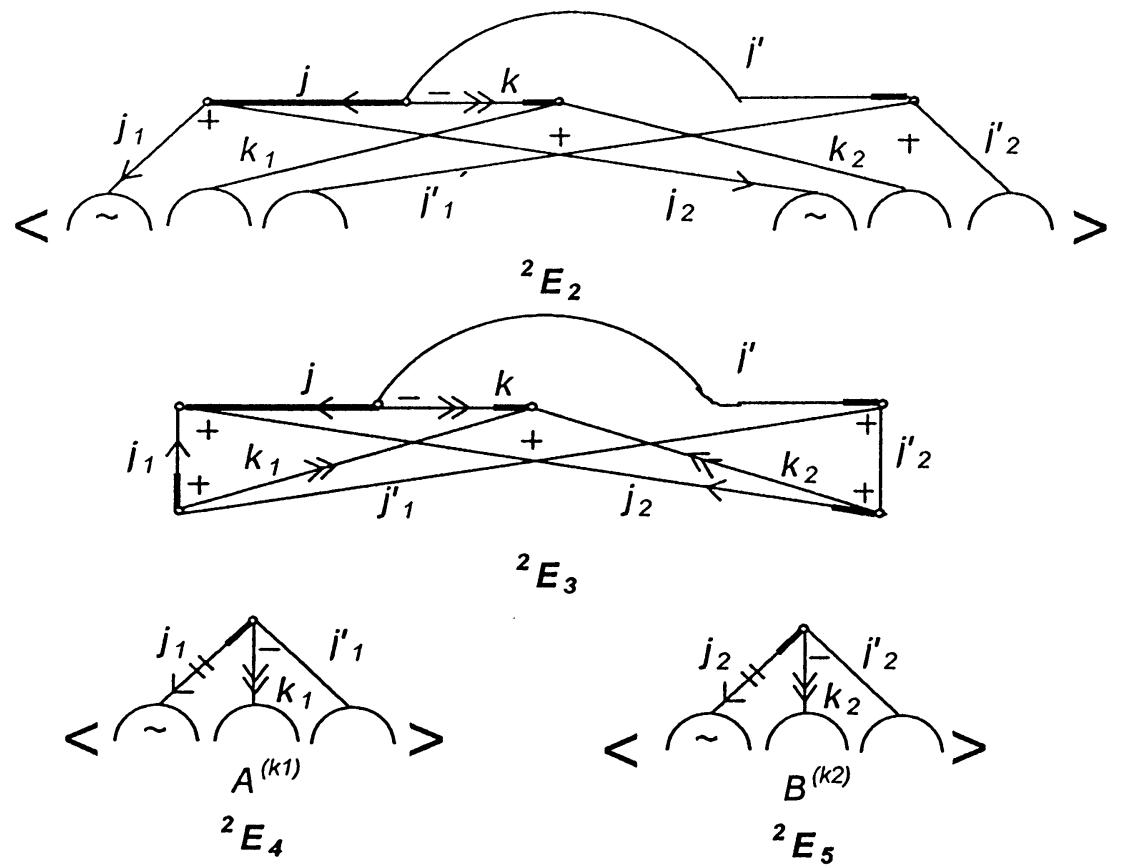

Fig. 8. Graphical evaluation of the submatrix element of the operator $\left[A^{\left(k_{1}\right)} \times B^{\left(k_{2}\right)}\right]^{(k)}$.

erators $[14,16]$. Namely, the operator $a_{m_{\lambda}}^{(\lambda)}=a_{\lambda m_{\lambda}}$ is connected with the diagram ${ }^{2} F_{1}$ while the operator

$$
\widetilde{a}_{m_{\lambda}}^{(\lambda)}=(-1)^{\lambda-m_{\lambda}} a_{\lambda-m_{\lambda}}^{+}
$$

is presented by ${ }^{2} F_{2}$. Here we use the abbreviations: $\lambda \equiv l s, m_{\lambda} \equiv m_{l} m_{s}$ for $L S$-coupling and $\lambda \equiv j$, $m_{\lambda} \equiv m_{j_{i}}$ for $j j$-coupling. By using the graphical relation $(\mathrm{g})$ of Fig. 6, we can depict the operation of the Hermitian conjugation applied to the operators $a_{m_{\lambda}}^{(\lambda)}$ and $\widetilde{a}_{m_{\lambda}}^{(\lambda)}$ (see the graphical relations (a) and (b) of Fig. 7). For instance, the graphical equation (b) represents the relationship $\left(\widetilde{a}_{m_{\lambda}}^{(\lambda)}\right)^{+}=$ $(-1)^{\lambda-m_{\lambda}} a_{-m_{\lambda}}^{(\lambda)}$. Following the given definitions, the Hermitian conjugation of complex tensorial products can be easily found [59]. For example, referring to (a) and (b) of Fig. 7 and the results of [53], we obtain the graphical relation (c) which imitates the expression [16]

$$
\begin{aligned}
& \left(\left[a^{\left(\lambda_{1}\right)} \times \widetilde{a}^{\left(\lambda_{2}\right)}\right]_{m_{\gamma}}^{(\gamma)}\right)^{+} \\
& \quad=(-1)^{\lambda_{1}-\lambda_{2}+m_{\gamma}}\left[a^{\left(\lambda_{2}\right)} \times \widetilde{a}^{\left(\lambda_{1}\right)}\right]_{-m_{\gamma}}^{(\gamma)} .
\end{aligned}
$$

In [53] one can find the graphical formulation and practical application of the Wick's theorem when electron creation and annihilation operators are presented by the irreducible tensorial products.

The above-listed conventions and definitions for the irreducible tensors will be used in the next sections for the graphical representation of $N$-electron operators and matrix elements.

\section{Graphical representation of $N$-particle operators}

The interactions in an atom are described by oneand two-particle operators. However, when applying the perturbation theory (PT), the effective operators corresponding to $N$-particle operators with $N>2$ 
appear. The generation of MBPT expansion terms is considered as the task of construction of some "effective" operator instead of sums of the products of matrix elements which appear in the expansions of PT $[14,35,36,45-47]$. In the present section we shall briefly consider the ways of constructing and the forms of one- and two-particle operators as well as particular effective operators used in graphical evaluation of various quantities of atomic spectroscopy.

In MBPT considerations (e.g., [48]) the Goldstone diagram ${ }^{3} A_{1}$ is associated with a one-particle operator

$$
F=\sum_{\alpha=1}^{N} f_{\alpha}=\sum_{\alpha=1}^{N} f\left(r_{\alpha}\right) f_{\alpha}^{(\gamma)} .
$$

Suppose that for the $L S J$ coupling scheme

$$
f=\sum_{m_{k} m_{\kappa}}\left[\begin{array}{ccc}
k & \kappa & \Gamma \\
m_{k} & m_{\kappa} & m_{\Gamma}
\end{array}\right] f(r) f_{m_{k} m_{\kappa}}^{(k \kappa)}=f(r) f_{m_{\Gamma}}^{(\gamma) \Gamma},
$$

and for the $L S$ one

$$
f=f(r) f^{(\gamma)}{ }_{m_{\gamma}} .
$$

Here $f(r)$ is the radial part of the operator $f$. The $f^{(k \kappa)}$ is an irreducible tensorial operator of ranks $k$ and $\kappa$ in the $l$ - and $s$-space. We have used the abbreviations $\gamma \equiv k \kappa, m_{\gamma} \equiv m_{k} m_{\kappa}$. In this paper we shall use $f$ in the form (34). Converting the obtained expression into the case (33) is trivial [58]. Below in this section, for brevity, we shall use the following notations: $\bar{i} \equiv n_{i} \lambda_{i} m_{i}$ and $i \equiv n_{i} \lambda_{i}$. Then, the operator $F$ in the second quantization form is given by [14]

$$
F=\sum_{\bar{i}, \bar{j}} a_{\bar{i}} a_{\bar{j}}\left(\bar{i}\left|f^{(\gamma)}\right| \bar{j}\right),
$$

where $\left(\bar{i}\left|f^{(\gamma)}\right| \bar{j}\right)$ is a matrix element of $f^{(\gamma)}$. The directed lines $\bar{i}$ and $\bar{j}$ of ${ }^{3} A_{1}$ are connected with $a_{\bar{i}}$ and $a_{j}^{+}$operators, respectively. The vertex of this diagram describes the matrix element of $f^{(\gamma)}$. The diagram ${ }^{3} A_{1}$ gives each term of Eq. (35) and depends on the magnetic quantum numbers $m_{i}, m_{j}$, and $m_{\gamma}$. When using the diagram ${ }^{3} A_{1}$ in regular evaluations $[14,33,36,46,48]$, the angular momentum part of ${ }^{3} A_{1}$ is associated with the graph ${ }^{3} A_{2}$ for the CGC which appears after the application of the Wigner-Eckart theorem (21) to $\left(\bar{i}\left|f^{(\gamma)}\right| \bar{j}\right)$ :

$$
F=\sum_{\bar{i}, \bar{j}} a_{\bar{i}} a_{\frac{j}{j}}^{ \pm}\left[i\left\|f^{(\gamma)}\right\| j\right]^{3} A_{2} .
$$

The operators $a_{\bar{i}}$ and $a_{\bar{j}}^{+}$are not explicitly involved in the graphical representation of $F$.
Similarly, the Goldstone diagram ${ }^{3} B_{1}$ is associated with a two-particle operator given in SQR [14]

$$
G=\frac{1}{2} \sum_{\bar{i}, \bar{j}, \overline{i^{\prime}}, \overline{j^{\prime}}} a_{\bar{i}} a_{\bar{j}} a_{\bar{j}^{\prime}}^{+} a_{\bar{i}^{\prime}}^{ \pm}\left(\overline{i j}\left|g^{\left(\gamma_{1}, \gamma_{2}\right)(\gamma)}{ }_{m_{\Gamma}}\right| \overline{i^{\prime} j^{\prime}}\right)
$$

or in the CR [46] when the tensorial structure of $g$ is shown explicitly, i. e.

$$
G=\sum_{\alpha<\beta}^{N} g_{\alpha \beta}=\sum_{\alpha<\beta}^{N} g\left(r_{\alpha}, r_{\beta}\right)\left[g_{\alpha}^{\left(\gamma_{1}\right)} \times g_{\beta}^{\left(\gamma_{2}\right)}\right]_{m_{\Gamma}}^{(\gamma)} .
$$

Analogically to the one-particle operator, after the application of the Wigner-Eckart theorem to the matrix element $\left(\overline{i j}\left|g^{\left(\gamma_{1}, \gamma_{2}\right)(\gamma)} m_{\Gamma}\right| \overline{i^{\prime} j^{\prime}}\right)$, one can obtain

$$
\begin{aligned}
G= & \frac{1}{2} \sum_{\bar{i}, \bar{j}, \overline{i^{\prime}}, \overline{j^{\prime}}} a_{\bar{i}} a \overline{\bar{j}} a \frac{+}{\bar{j}^{\prime}} a a_{\bar{i}^{\prime}}^{+} \\
& \times\left[i j\left\|g^{\left(\gamma_{1}, \gamma_{2}\right)(\gamma)}{ }_{m_{\Gamma}}\right\| i^{\prime} j^{\prime}\right]^{3} B_{2}\left(\bar{i}, \bar{j}, \overline{i^{\prime}}, \overline{j^{\prime}}\right),
\end{aligned}
$$

where the diagram ${ }^{3} B_{2}\left(\bar{i}, \bar{j}, \overline{i^{\prime}}, \overline{j^{\prime}}\right)$ represents a sum of the products of three CGCs. Note that when treating $G$ in $L S J$ coupling, the GCG of (33) has to be involved [58]. In PT studies of $[14,35,36,48]$ the operators $F$ and $G$ are explored in the forms (36) and (39), however, only the graphs ${ }^{3} A_{2}$ and ${ }^{3} B_{2}$ are used to construct the momentum part of a composite operator. The remaining quantities of Eqs. (36) and (39) were involved in algebraic manipulations with $F$ and $G$.

In the approach of $[52,53,58,59]$, the tensorial properties of the electron creation and annihilation operators are used to develop the diagrams which explicitly involve these operators in the representation of $N$-electron operators and many-electron states. For instance, the right-hand side of Eq. (35) is presented by the sum of products of the diagrams ${ }^{2} F_{1},{ }^{2} F_{3}$, and ${ }^{2} D_{4}$. The matrix element $\left(\bar{i}\left|f^{(\gamma)}\right| \bar{j}\right)$ is associated with the diagram ${ }^{2} D_{4}$ while the operators $a_{\bar{i}}$ and $a_{\bar{j}}^{+}$are given by ${ }^{2} F_{1}$ and ${ }^{2} F_{3}$, respectively. Let us assume at the moment that the radial integral $\left(n_{i} l_{i}|f(r)| n_{j} l_{j}\right)$ is included into ${ }^{2} D_{4}$. The sum of these diagrams over $m_{i}$ and $m_{j}$ gives ${ }^{3} A_{3}$ which can be decomposed in the following way [59]:

$$
F=\sum_{i j}{ }^{3} A_{3}=\sum_{i j}{ }^{3} A_{4} M C\left(\lambda_{i}, \lambda_{j}, f^{(\gamma)}\right) .
$$

The quantity $M C\left(\lambda_{i}, \lambda_{j}, f^{(\gamma)}\right)$ (see the graphical relation (b) of Fig. 9) corresponds to the vertex of ${ }^{3} A_{3}$ and is referred to as a "marking circle" (following [44]). After elimination of $M C\left(\lambda_{i}, \lambda_{j}, f^{(\gamma)}\right)$ from the diagram ${ }^{3} A_{3}$ (this is described by (c) of Fig. 9) it transforms to the diagram ${ }^{3} A_{4}$ which denotes the irreducible 


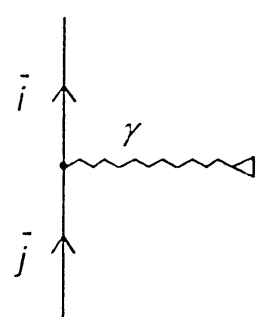

${ }^{3} A_{1}$

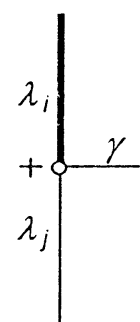

${ }^{3} \mathrm{~A}_{2}$ a)

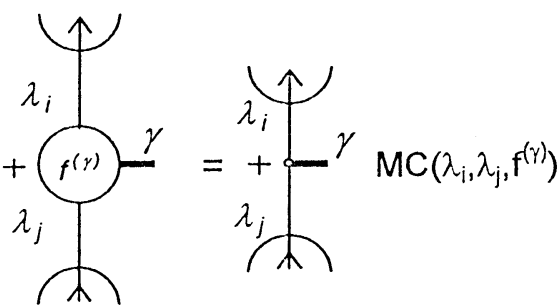

${ }^{3} \mathrm{~A}_{3}$ b)

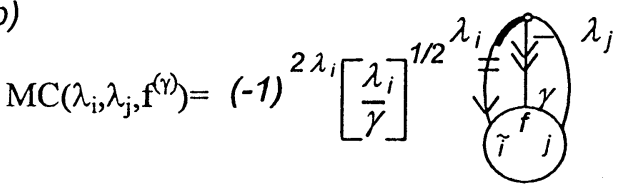

${ }^{3} \mathrm{~A}_{5}$ c)

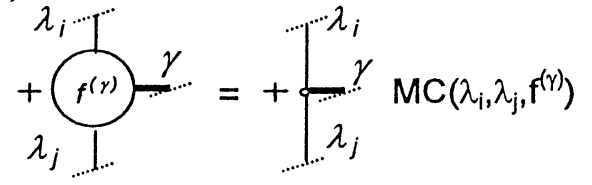

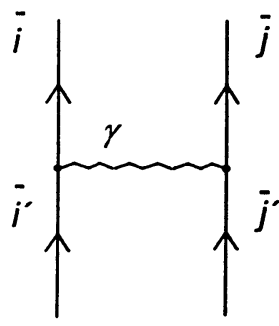

${ }^{3} B_{1}$

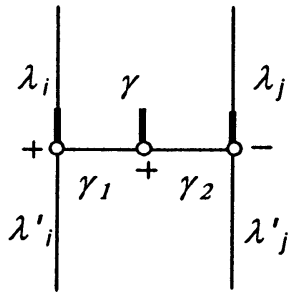

${ }^{3} B_{2}$

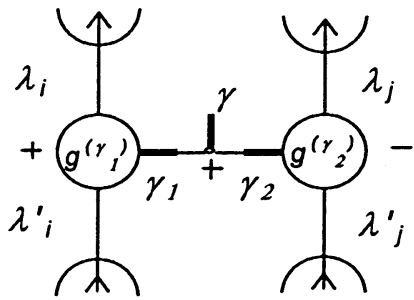

${ }^{3} B_{3}$

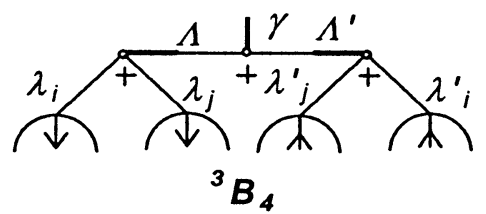

Fig. 9. Diagrams for one- and two-particle operators.

tensorial product $\left[a^{\left(\lambda_{i}\right)} \times \widetilde{a}^{\left(\lambda_{j}\right)}\right]_{m_{\gamma}}^{(\gamma)}$. The graph ${ }^{3} A_{5}$ denotes SBE $\left[\lambda_{i}\|f(\gamma)\| \lambda_{j}\right]$. Thus, the diagram ${ }^{3} A_{3}$ represents the operator $F$ with the rank $\gamma$ and projection $m_{\gamma}$ in the coupled form and is independent of the oneelectron magnetic quantum numbers $m_{\lambda_{i}}$.

Following the way just described, we can easily obtain the following expression for the two-particle operator:

$$
G=\sum_{i j i^{\prime} j^{\prime}}{ }^{3} B_{3} .
$$

The diagram ${ }^{3} B_{3}$ yields the coupled form of a two- particle operator $G$. Here we assume that the radial part $\left(n_{i} l_{i} n_{j} l_{j}\left|g\left(r_{1}, r_{2}\right)\right| n_{i}^{\prime} l_{i}^{\prime} n_{j}^{\prime} l_{j}^{\prime}\right)$ is included in ${ }^{3} B_{3}$. The final graphical representation for the operator $G$ depends not only on the coupling scheme for momenta but also on the order in which the operators $a^{(\lambda)}$ and $\widetilde{a}^{(\lambda)}$ are presented in an expression for $G$. The diagram ${ }^{3} B_{3}$ represents an expression where operators $a^{(\lambda)}$ and $\widetilde{a}^{(\lambda)}$ are given in the order $a^{\left(\lambda_{i}\right)} a^{\left(\lambda_{j}\right)} \widetilde{a}^{\left(\lambda_{j}^{\prime}\right)} \widetilde{a}^{\left(\lambda_{i}^{\prime}\right)}$ (normal ordering [48]). The coupling scheme is such that the ranks $\lambda_{i}\left(\lambda_{j}\right)$ and $\lambda_{i}^{\prime}\left(\lambda_{j}^{\prime}\right)$ are coupled to the resultant rank $\gamma_{1}\left(\gamma_{2}\right)$. To obtain the algebraic expression for the operator $G$ in the normal ordering, one needs to change 
the coupling scheme, where $\lambda_{i}\left(\lambda_{j}^{\prime}\right)$ is coupled with $\lambda_{j}$ $\left(\lambda_{i}^{\prime}\right)$ to $\Lambda\left(\Lambda^{\prime}\right)$. By using for this the GCGC ${ }^{1} B_{2}$ we obtain

$$
\begin{aligned}
G & =\sum_{i j i^{\prime} j^{\prime}}{ }^{3} B_{3} \\
& =\frac{1}{2} \sum_{i j i^{\prime} j^{\prime} \Lambda \Lambda^{\prime}}(-1)^{\lambda_{i}^{\prime}+\lambda_{j}^{\prime}-\Lambda^{\prime}}\left[\frac{\Lambda}{\gamma}\right]^{1 / 2}{ }^{3} B_{4}{ }^{2} E_{1} .
\end{aligned}
$$

Here for ${ }^{2} E_{1}$ the following substitutions have to be done: $j=\Lambda, j^{\prime}=\Lambda^{\prime}, \gamma=k$ and $j_{i}=\lambda_{i}$, $k_{i}=\gamma_{i}(i=1,2), j_{1}^{\prime}=\lambda_{j}^{\prime}$ and $j_{2}^{\prime}=\lambda_{i}^{\prime}$. The diagram ${ }^{3} B_{4}$ represents the irreducible tensorial product

$$
{ }^{3} B_{4}=\left[\left[a^{\left(\lambda_{i}\right)} \times a^{\left(\lambda_{j}\right)}\right]^{(\Lambda)} \times\left[\widetilde{a}^{\left(\lambda_{j}^{\prime}\right)} \times \widetilde{a}^{\left(\lambda_{i}^{\prime}\right)}\right]^{\left(\Lambda^{\prime}\right)}\right]^{(\gamma)} .
$$

Frequently, the operator $G$ is used with the antisymmetric amplitude [14], i.e. when the matrix element $\left(\overline{i j} \mid g^{\left(\gamma_{1}, \gamma_{2}\right)(\gamma)} m_{\Gamma} \overline{i^{\prime} j^{\prime}}\right)$ in Eq. (37) is determined by the antisymmetric two-electron wave functions. Note that even in earlier times a general expression of the matrix element for the scalar operator $g^{\left(\gamma_{1}, \gamma_{2}\right)(\gamma)}$ in the case of the antisymmetric two-electron wave fuctions was obtained in [26] by using the graphical approach of [1]. In SQR one can write [60]

$$
\begin{aligned}
G= & \frac{1}{4} \sum_{i j i^{\prime} j^{\prime} \Lambda \Lambda^{\prime}}(-1)^{\lambda_{i}^{\prime}+\lambda_{j}^{\prime}-\Lambda^{\prime}}\left[\frac{\Lambda}{\gamma}\right]^{1 / 2} \\
& \times{ }^{3} B_{4}\left\{\left[1-(-1)^{\lambda_{i}^{\prime}+\lambda_{j}^{\prime}-\Lambda^{\prime}} P\left(\lambda_{i}^{\prime}, \lambda_{j}^{\prime}\right)\right]\right\}^{2} E_{1},
\end{aligned}
$$

where $P(a, b)$ is the operator or permutation of quantities $a$ and $b$.

Let us return now to the study of diagrammatic approach of PT considering this problem as the task of obtaining the product of operators given in SQR. The Wick's theorem is used for this purpose [48]. We do not go into details of PT, but restrict our interest to a construction of angular-momentum part of the Goldstone diagram. For example, consider the diagram ${ }^{3} C_{1}$ of the second-order stationary PT [48]. The diagram ${ }^{3} C_{1}$ is obtained as a particular term of the Wick's product of two diagrams of the type ${ }^{3} B_{1}$, when two pairs of electron creation and annihilation operators are contracted. In the diagrammatic language these contractions are presented by directed $t$ and $p$ lines in ${ }^{3} C_{1}$. By using regular rules of PT to write an algebraic expression for the Goldstone diagram (see, for example, [48]), it is obtained

$$
\begin{aligned}
\Theta & \equiv \sum_{\bar{i}, \bar{j}, \overline{i^{\prime}}, \overline{j^{\prime}}, \bar{t}, \bar{p}}{ }^{3} C_{1} \\
& =\frac{1}{2} \sum_{\bar{i}, \bar{j}, \overline{i^{\prime}}, \overline{j^{\prime}}, \bar{t}, \bar{p}} a_{\bar{i}} a \overline{\bar{j}} a \frac{+}{\bar{j}^{\prime}} a \frac{\left\langle\overline{i j}\left|C_{2}\right| \bar{t} \bar{p}\right\rangle\left\langle\left.\bar{t} \bar{p}\left|C_{1}\right|\right|^{\prime} \bar{j}^{\prime}\right\rangle}{\varepsilon_{j^{\prime}}+\varepsilon_{i^{\prime}}-\varepsilon_{t}-\varepsilon_{p}} .
\end{aligned}
$$

In Eq. (45) $\left\langle\overline{i j}\left|C_{u}\right| \bar{t} \bar{p}\right\rangle$ represents the matrix element of a scalar two-particle operator $G$ when $\gamma=0 . \varepsilon_{i}$ denotes the single-particle eigenvalue associated with the orbital $\left.\mid n_{i} \lambda_{i} m_{i}\right)$. We now follow the way of [14] and [48] to detect the momentum part of ${ }^{3} C_{1}$. Note that in the mentioned papers only the case of one open shell $\left(i=j=i^{\prime}=j^{\prime}\right)$ for the Coulomb interaction is discussed. By using the operator $G$ in the form (39) and by applying the summation rule, it was found

$$
\begin{aligned}
\Theta= & \frac{1}{2} \sum_{\bar{i}, \bar{j}, \overline{i^{\prime}}, \bar{j}^{\prime} t, p, k_{1}, k_{2}} a_{\bar{i}} a_{\bar{j}} a_{\overline{j^{\prime}}} a_{i^{\prime}}^{ \pm} \\
& \times \frac{X\left(k_{2}, i, j, t, p\right) X\left(k_{1}, t, p, i^{\prime}, j^{\prime}\right)}{\varepsilon_{j^{\prime}}+\varepsilon_{i^{\prime}}-\varepsilon_{t}-\varepsilon_{p}} C_{2},
\end{aligned}
$$

where

$$
\begin{aligned}
& X\left(k, i, j, i^{\prime}, j^{\prime}\right) \\
& =(-1)^{k}\left(l_{i}\left\|C^{(k)}\right\| l_{i}^{\prime}\right)\left(l_{j}\left\|C^{(k)}\right\| l_{j}^{\prime}\right) \\
& \quad \times R_{k}\left(n_{i} l_{i} n_{j} l_{j}, n_{i}^{\prime} l_{i}^{\prime} n_{j}^{\prime} l_{j}^{\prime}\right) .
\end{aligned}
$$

Here $R_{k}\left(n_{i} l_{i} n_{j} l_{j}, n_{i}^{\prime} l_{i}^{\prime} n_{j}^{\prime} l_{j}^{\prime}\right)$ is the general radial integral for the Coulomb interaction, $\left(l_{i}\left\|C^{(k)}\right\| l_{i}^{\prime}\right)$ is the reduced matrix element of the spherical function $C^{(k)}$. In the approach of [48], the diagram ${ }^{3} C_{2}$ is composed from the WC. Let us emphasize that the topological equivalence between the diagrams ${ }^{3} C_{1}$ and ${ }^{3} C_{2}$ was the cardinal result of [33] which encouraged researchers for the subsequent investigations in this direction. Note that in [33] only the closed-shell case for the Coulomb interaction was considered. By using the JLV Theorem $2,{ }^{3} C_{2}$ was transformed into the form [48]:

$$
{ }^{3} C_{2}=\sum_{k} E E(k){ }^{3} C_{3}\left(\bar{i}, \bar{j}, \overline{i^{\prime}}, \overline{j^{\prime}}\right),
$$

where the $\mathrm{RC}$ is

$$
E E(k)=(-1)^{k}[k]\left\{\begin{array}{ccc}
l & l & k \\
k_{1} & k_{2} & l_{t}
\end{array}\right\}\left\{\begin{array}{ccc}
l & l & k \\
k_{1} & k_{2} & l_{p}
\end{array}\right\} .
$$


The special coupling scheme of ${ }^{3} C_{3}\left(\bar{i}, \bar{j}, \overline{i^{\prime}}, \overline{j^{\prime}}\right)$ and the relation [48]

$$
\begin{aligned}
& \sum_{\alpha<\beta}^{N}\left(u^{(k 0)}(\alpha) u^{(k 0)}(\beta)\right) \\
& \quad=\frac{1}{2} \sum_{\overline{i j i^{\prime} j^{\prime}}}(-1)^{k} a_{\bar{i}} a \bar{j} a \frac{ \pm}{\bar{j}^{\prime}} a_{{\overline{i^{\prime}}}^{\prime}}^{+3} C_{3}\left(\overline{i, j, \bar{i}^{\prime}}, \overline{j^{\prime}}\right)
\end{aligned}
$$

allow one to replace the operator given in $\mathrm{SQR}$ in the right-hand side of Eq. (46) to the one expressed in CR. Here $u^{(k 0)}$ is the operator with the orbital rank equal to $k$ whereas the rank of $s$-space is zero [14]. Then,

$$
\begin{aligned}
\Theta= & \sum_{\substack{\alpha<\beta, k_{1} k_{2} k t p i j i^{\prime} j^{\prime}}}^{N}\left[\left(u^{(k 0)}(\alpha) u^{(k 0)}(\beta)\right) E E(k)\right] \\
& \times \frac{X\left(k_{2}, i, j, t, p\right) X\left(k_{1}, t, p, i^{\prime}, j^{\prime}\right)}{\varepsilon_{j^{\prime}}+\varepsilon_{i^{\prime}}-\varepsilon_{t}-\varepsilon_{p}} .
\end{aligned}
$$

Thus, to obtain the final form for a PT expansion term in addition to graphical procedures one has to use some algebraic manipulations (of the type (50)) which allow one to form the effective operator in the CR. However, this is not a trivial task when treating atoms with several open shells and searching for optimal expressions.

The approach of $[52,58,59]$ gives additional possibilities while the formation of the expressions for PT expansion terms and the derivation are carried out purely graphically. To illustrate this, again consider the diagram ${ }^{3} C_{1}$. In contrast to the previous studies suppose that ${ }^{3} C_{1}$ can operate on several shells of equivalent electrons and $g^{\left(\gamma_{1}, \gamma_{2}\right)(\gamma)}$ is a scalar operator but not necessarily the operator of the Coulomb interaction. Then we can write

$$
\Theta=\frac{1}{2} \sum_{i j i^{\prime} j^{\prime} t p \gamma_{1} \gamma_{2}}{ }^{3} D_{1} .
$$

The diagram ${ }^{3} D_{1}$ is obtained in a very simple way from the Goldstone diagram ${ }^{3} C_{1}$ by using the following rules [58, 59]:

(1) the free lines of the diagram ${ }^{3} C_{1}$ associated with creation and annihilation operators are to be replaced by the corresponding graphical elements ${ }^{2} F_{1}$ and ${ }^{2} F_{2}$, respectively;

(2) the internal electronic line $t$ of ${ }^{3} C_{1}$ has to be replaced by the momentum line $\lambda_{t}$ of the same direction as the line $t$;
(3) each node of ${ }^{3} C_{1}$ has to be replaced by the marking circle associated with the corresponding operator $g^{\left(\gamma_{u}\right)}$ of $g^{\left(\gamma_{1}, \gamma_{2}\right)(\gamma)}$

(4) each dotted line has to be replaced by GCGC which represents the coupling scheme of $g^{\left(\gamma_{1}, \gamma_{2}\right)(\gamma)}$.

The rules given above are valid for the construction of an angular-momentum diagram for an arbitrary diagram of PT not only for the diagram ${ }^{3} C_{1}$. The weight, phase, and radial factors of the diagram ${ }^{3} C_{1}$ are determined by the rules given in the textbooks of PT (see, for instance, [48]). Thus, the MBPT diagram ${ }^{3} C_{1}$ is connected with the graph ${ }^{3} D_{1}$ which represents the PT expansion term as an effective operator of a coupled form, i. e. this term does not depend on the one-electron magnetic quantum numbers $m$. By using the rules for transformation of irreducible tensorial products given in Sections 1 and 2, the diagram ${ }^{3} D_{1}$ can be given in the following form [57]:

$$
\begin{aligned}
{ }^{3} D_{1}= & \sum_{x}{ }^{3} D_{2}(x){ }^{3} D_{3}(x) \\
& \times \frac{\bar{X}\left(\gamma_{2}, i, j, t, p\right) \bar{X}\left(\gamma_{1}, t, p, i^{\prime}, j^{\prime}\right)}{\varepsilon_{j^{\prime}}+\varepsilon_{i^{\prime}}-\varepsilon_{t}-\varepsilon_{p}},
\end{aligned}
$$

where

$$
\begin{aligned}
\bar{X}\left(\gamma, i, j, i^{\prime}, j^{\prime}\right) \\
=M C\left(\lambda_{i}, \lambda_{i^{\prime}}, g^{(\gamma)}\right) M C\left(\lambda_{j}, \lambda_{j^{\prime}}, g^{(\gamma)}\right) \\
\quad \times\left(n_{i} l_{i} n_{j} l_{j}\left|g\left(r_{1}, r_{2}\right)\right| n_{i}^{\prime} l_{i}^{\prime} n_{j}^{\prime} l_{j}^{\prime}\right),
\end{aligned}
$$

and the RC

$$
\begin{aligned}
{ }^{3} D_{3}(x)= & \sum_{x}(-1)^{\gamma_{1}+\gamma_{2}+\lambda_{j}-\lambda_{j}^{\prime}+\lambda_{t}+\lambda_{p}+x}\left[\gamma_{1}, \gamma_{2}, x\right]^{1 / 2} \\
& \times\left\{\begin{array}{c}
\lambda_{t} \lambda_{i} \gamma_{2} \\
\lambda_{j} \lambda_{p} x
\end{array}\right\}\left\{\begin{array}{l}
\lambda_{t} \lambda_{i}^{\prime} \gamma_{1} \\
\lambda_{j}^{\prime} \lambda_{p} x
\end{array}\right\} .
\end{aligned}
$$

The diagram ${ }^{3} D_{2}$ represents the irreducible tensorial product with the resulting rank equal to zero, i.e. ${ }^{3} D_{2}(x)={ }^{3} B_{4}$ when $\gamma=0$. While formatting this diagram, the graph ${ }^{1} B_{7}$ was used. When the operator ${ }^{3} D_{1}$ acts on one shell of the equivalent electrons and $\gamma=k 0$, the expression (53) for ${ }^{3} D_{1}$ corresponds to (51). Note that when ${ }^{3} D_{1}$ acts on several shells, the tensorial structure ${ }^{3} D_{2}$ of the operator ${ }^{3} D_{1}$ is not the most optimal. Assume that ${ }^{3} D_{1}$ acts on two open shells, for example, $i=j^{\prime}=$ $n_{1} \lambda_{1}$ and $j=i^{\prime}=n_{2} \lambda_{2}$. Then the expression for the SBE of ${ }^{3} D_{2}$ contains the sum over the quantum numbers of the intermediate angular momenta. 
The operator of the desired form (then the expression for SBE is given without intermediate summation) can be very easily obtained from ${ }^{3} D_{1}$ in the graphical way as it has been done by deriving ${ }^{3} D_{2}$. For this one should choose the coupling scheme $B=$ $\left\{i j^{\prime}(x), j i^{\prime}(x), 0\right\}$ of Section 1. In this case the tensor

$$
\left[\left[a^{\left(\lambda_{i}\right)} \times \widetilde{a}^{\left(\lambda_{j}^{\prime}\right)}\right]^{(x)} \times\left[a^{\left(\lambda_{j}\right)} \times \widetilde{a}^{\left(\lambda_{i}^{\prime}\right)}\right]^{(x)}\right]^{(0)}
$$

is obtained. The expression of the SBE for this tensor can be found from Eq. (29) by using $\left[a^{\left(\lambda_{i}\right)} \times \widetilde{a}^{\left(\lambda_{j}^{\prime}\right)}\right]^{(x)}$ and $\left[a^{\left(\lambda_{j}\right)} \times \widetilde{a}^{\left(\lambda_{i}^{\prime}\right)}\right]^{(x)}$ instead of $A^{\left(k_{1}\right)}$ and $B^{\left(k_{2}\right)}$, respectively, and by putting $k=0$ in Eq. (29). For more details of such derivation, see the following section.

Generalizing the study of the diagram ${ }^{3} C_{1}$ we can conclude that the sum of an arbitrary Goldstone diagram over the one-electron magnetic quantum numbers $m$ is carried out in a very simple way: one has to replace each element of the Goldstone diagram by the corresponding element of the graphical approach discussed by using the rules given below (52). Actually, the obtained diagram is topologically equivalent to the Goldstone diagram. The operators of atomic interactions and the perturbation expansion terms are given by diagrams which correspond to the irreducible tensorial products composed of $a^{(\lambda)}$ and $\widetilde{a}^{(\lambda)}$. By using the graphical procedures of Sections 1 and 2, these diagrams can be expressed by other diagrams (corresponding to the operators with the less complex expressions for matrix elements) prior to the study of matrix elements. Thus, we have the possibility to reduce the composed operator by singling out from it the operators of the type $N, L^{2}, S^{2}$, etc. which have very simple expressions for the matrix elements $[59,60]$. All manipulations are done in a purely graphical way.

The method of the construction of an effective operator of PT can also be applied not only to MBPT but to some other problems of atomic spectroscopy $[60,67]$. For instance, the transition probabilities or cross-sections of scattering problems are described by the quantities of type $\left|\left[\alpha_{1} j_{1} m_{1}\left|T_{q}^{(k)}\right| \alpha_{2} j_{2} m_{2}\right]\right|^{2}$ summed over some of the quantum numbers $\alpha_{i}, j_{i}, m_{i}$ and $k, q$ depending on the problem considered. The underlying relation

$$
\begin{aligned}
& \sum_{\alpha_{2} j_{2} m_{2}}\left[\alpha_{1} j_{1} m_{1}\left|T_{q_{1}}^{\left(k_{1}\right)}\right| \alpha_{2} j_{2} m_{2}\right] \\
& \times\left[\alpha_{2} j_{2} m_{2}\left|T_{q_{2}}^{\left(k_{2}\right)}\right| \alpha_{3} j_{3} m_{3}\right] \\
& =\sum_{\alpha_{2} j_{2} m_{2}}{ }^{3} E_{1}{ }^{3} E_{2}=\sum_{\alpha_{2} j_{2}}{ }^{3} E_{3}={ }^{3} E_{4} \\
& =\left[\alpha_{1} j_{1} m_{1}\left|T_{q_{1}}^{\left(k_{1}\right)} T_{q_{2}}^{\left(k_{2}\right)}\right| \alpha_{3} j_{3} m_{3}\right] \\
& =\left[\alpha_{1} j_{1} m_{1}\left|T_{\mathrm{eff}}\right| \alpha_{3} j_{3} m_{3}\right]
\end{aligned}
$$

is used to determine an effective operator. The product of the matrix elements of the operators $T_{q_{1}}^{\left(k_{1}\right)}$ and $T_{q_{2}}^{\left(k_{2}\right)}$ can be represented by the product of the corresponding diagrams ${ }^{3} E_{1}$ and ${ }^{3} E_{2}$ (see graphical relation (a) of Fig. 10). The summation over $m_{2}$ yields ${ }^{3} E_{3}$. Finally, the diagram ${ }^{3} E_{4}$ gives the matrix element of the product of $T_{q_{1}}^{\left(k_{1}\right)}$ and $T_{q_{2}}^{\left(k_{2}\right)}$. Suppose we study the parts ${ }_{s} T_{q_{1}}^{\left(k_{1}\right)}$ and ${ }_{s} T_{q_{2}}^{\left(k_{2}\right)}$ of $T_{q_{1}}^{\left(k_{1}\right)}$ and $T_{q_{2}}^{\left(k_{2}\right)}$ which have a nonzero matrix element only between the states of the selected wave functions $\left.\mid \alpha_{1} j_{1} m_{1}\right]$, $\left.\mid \alpha_{2} j_{2} m_{2}\right]$, and $\left.\mid \alpha_{3} j_{3} m_{3}\right]$ with fixed $\alpha_{i} j_{i}$ and arbitrary $m_{i}$, namely $\left[\alpha_{1} j_{1} m_{1}\left|{ }_{s} T_{q_{1}}^{\left(k_{1}\right)}\right| \alpha_{2} j_{2} m_{2}\right] \neq 0$ and $\left[\left.\alpha_{2} j_{2} m_{2}\right|_{s} T_{q_{2}}^{\left(k_{2}\right)} \mid \alpha_{3} j_{3} m_{3}\right] \neq 0$. In this case the effective operator

$$
T_{\text {eff }}={ }_{s} T_{q_{1}}^{\left(k_{1}\right)}{ }_{s} T_{q_{2}}^{\left(k_{2}\right)}
$$

instead of $T_{q_{1}}^{\left(k_{1}\right)} T_{q_{2}}^{\left(k_{2}\right)}$ can be used (see, for example, [14]). For the specific case of Eq. (56) we can write [60]:

$$
\begin{aligned}
& \sum_{\alpha_{2} j_{2} m_{2} q}\left|\left[\alpha_{1} j_{1} m_{1}\left|T_{q}^{(k)}\right| \alpha_{2} j_{2} m_{2}\right]\right|^{2} \\
& \quad=\sum_{\alpha_{2} j_{2} q}{ }^{3} E_{3}=(-1)^{2 k}[k]^{1 / 2}{ }^{3} E_{6} \\
& =(-1)^{2 k}[k]^{1 / 2}\left[\lambda_{1} m_{\lambda_{1}}\left|\left[{ }_{s} T^{(k)}{ }_{s} \widetilde{T}^{(k)}\right]_{0}^{(0)}\right| \lambda_{2} m_{\lambda_{2}}\right] .
\end{aligned}
$$

The diagram ${ }^{3} E_{6}$ gives the matrix element of the irreducible tensorial product $\left[{ }_{s} T^{(k)}{ }_{s} \widetilde{T}^{(k)}\right]^{(0)}$. In [60] the graphical way for the construction of the effective operators was described and some illustrations of such construction were presented: for the Coulomb interaction when $\alpha_{1} j_{1}=\lambda_{1}^{N_{1}} \lambda_{2}^{N_{2}} \lambda_{3}^{N_{3}}$ and $\alpha_{2} j_{2}=\lambda_{1}^{N_{1}+1} \lambda_{2}^{N_{2}-2} \lambda_{3}^{N_{3}+1}$ and for the spin-orbit interactions when $\alpha_{1} j_{1}=\lambda_{1}^{N_{1}} \lambda_{2}^{N_{2}}$ and $\alpha_{2} j_{2}=$ $\lambda_{1}^{N_{1}-1} \lambda_{2}^{N_{2}+1}$. The general expression for the width 


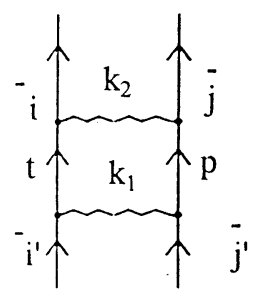

${ }^{3} C_{1}$

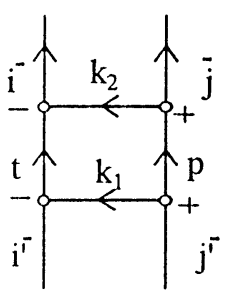

${ }^{3} C_{2}$

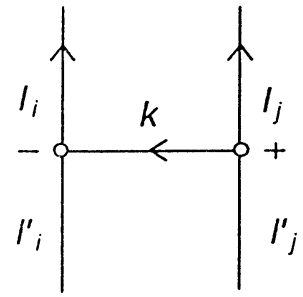

${ }^{3} \mathrm{C}_{3}$

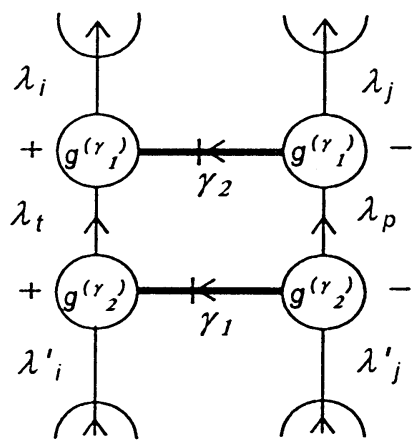

${ }^{3} D_{1}$

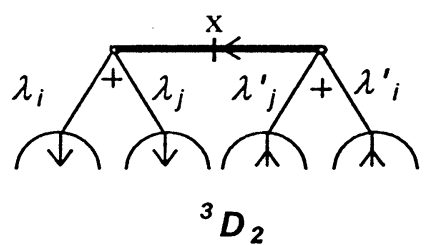

a)

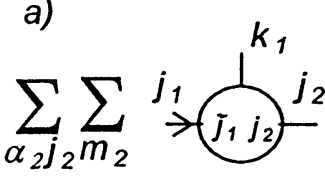

${ }^{3} E_{1}$

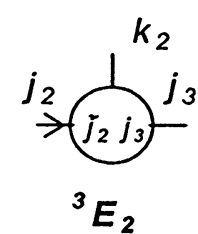

$=\sum_{\alpha, j_{2}}^{j_{1}} \rightarrow \overbrace{j_{1} j_{2}}^{k_{1}} \stackrel{j_{2}}{\longrightarrow} \overbrace{j_{2} j_{3}}^{k_{2}}=$

${ }^{3} E_{3}$

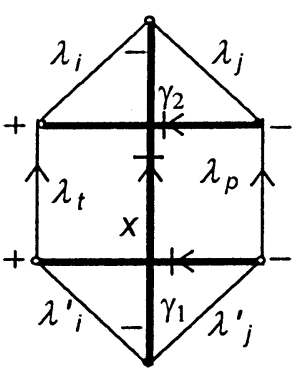

${ }^{3} D_{3}$<smiles>CCC(C)CC(C)CC(C)CC(CC)C(C)C</smiles>

${ }^{3} E_{4}$
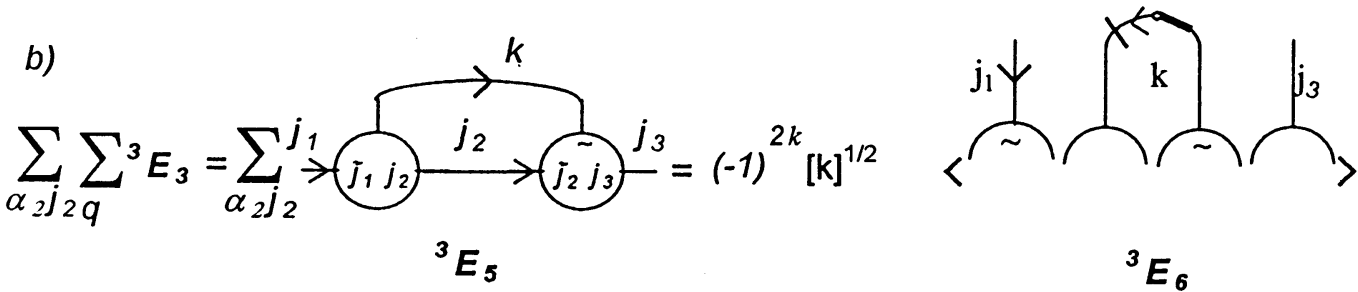

Fig. 10. Diagrammatic representation of effective operators.

of an energy level of an atom due to the Auger transitions was derived in [61] by using this graphical technique.

In the series of papers [71,72] the graphical approach of [31] was used to derive the expressions for the cross-sections of interaction of the polarized atoms with the polarized photons and electrons. The re- lations of the type (56), (58) were used when $T_{q}^{(k)}$ was the operator of the multipole radiation or the Coulomb interaction. The peculiarity of the study is to construct the general angular momentum diagram of the process considered which takes into account polarization and the angular dependence of the constituents of the process. Later on, the obtained 
complex diagram is decomposed in the way that polarization and angular dependence properties are described by the scalar tensorial product. The rest part of the diagram is presented as a multiple sum of products of RC and SBE characterizing interactions.

In $[67,68]$ the diagrammatic method for the calculation of vacuum expectation values for the products of operators was developed. The operators $F$ and $G$ were associated with the diagrams which were obtained by removing the wavy (interaction) line from ${ }^{3} A_{1}$ and ${ }^{3} B_{1}$. The vacuum expectation value is determined by the definite system of the contractions of $a_{\lambda m_{\lambda}}$ and $a_{\lambda m_{\lambda}}^{+}$which is presented by the diagram. We do not go into details of generation and classification of these diagrams but indicate the way the angularmomentum part of the diagram is obtained. The vertex of a diagram connected to the operator $F$ is replaced by the right-hand side of Eq. (36). The vertex of the diagram connected to the operator $G$ is replaced by the GCGC which is obtained when ${ }^{3} B_{4}$ is written in an uncoupled form in (44). The remaining quantities of (44) are considered as factors. In this way a closed diagram for orbital and spin momenta is constructed. Later on, these diagrams are reduced by using the JLV Theorems. The developed diagrammatic method was used to study global characteristics of energy levels and the Auger transitions [67].

\section{5. $N$-electron reduced matrix elements}

The graphical evaluation of matrix elements for many-electron case in addition to standard elements and rules of angular momentum theory needs the graphical elements and rules which reflect the antisymmetric properties of an $N$-electron wave function. In the algebraic approach when a shell of equivalent electrons is considered, the coefficient of fractional parentage is used for this purpose. The antisymmetrization operator ensures antisymmetry of the wave function between shells (see, for example, [69,70]). These two aspects were explored slightly differently in various graphical techniques [35-37, 39-42, 44, 47].

The regular way of the consideration is based on the following relations for one- and two-electron coefficients of fractional parentage [12]. One-particle CFP $\left(\lambda^{N} \alpha \Lambda \| \lambda^{N-1}\left(\alpha^{\prime} \Lambda^{\prime}\right) \lambda\right)$ is defined by

$$
\begin{aligned}
& \left|n \lambda^{N} \alpha \Lambda M_{\Lambda}\right\rangle \\
& =\sum_{\alpha^{\prime} \Lambda^{\prime} M_{\Lambda}^{\prime} m_{\lambda}}\left|n \lambda^{N-1} \alpha^{\prime} \Lambda^{\prime} M_{\Lambda}^{\prime}\right\rangle\left|n \lambda m_{\lambda}\right\rangle \\
& \quad \times\left(\lambda^{N} \alpha \Lambda \| \lambda^{N-1}\left(\alpha^{\prime} \Lambda^{\prime}\right) \lambda\right)\left[\begin{array}{ccc}
\Lambda^{\prime} & \lambda & \Lambda \\
M_{\Lambda}^{\prime} m_{\lambda} & M_{\Lambda}
\end{array}\right] .
\end{aligned}
$$

$\left|n \lambda^{N} \alpha \Lambda M_{\Lambda}\right\rangle$ denotes the bra-state for the shell $n \lambda$ of $N$ equivalent electrons. Similarly, a two-particle CFP $\left(\lambda^{N} \alpha \Lambda \| \lambda^{N-2}\left(\alpha^{\prime} \Lambda^{\prime}\right), \lambda^{2}\left(\Lambda_{0}\right)\right)$ is defined by

$$
\begin{aligned}
& \left|n \lambda^{N} \alpha \Lambda M_{\Lambda}\right\rangle \\
& =\sum_{\alpha^{\prime} \Lambda^{\prime} M_{\Lambda}^{\prime} \Lambda_{0} M_{\Lambda_{0}}}\left|n \lambda^{N-2} \alpha^{\prime} \Lambda^{\prime} M_{\Lambda}^{\prime}\right\rangle\left|n \lambda^{2} \Lambda_{0} M_{\Lambda_{0}}\right\rangle \\
& \quad \times\left(\lambda^{N} \alpha \Lambda \| \lambda^{N-2}\left(\alpha^{\prime} \Lambda^{\prime}\right) \lambda^{2}\left(\Lambda_{0}\right)\right) \\
& \quad \times\left[\begin{array}{ccc}
\Lambda^{\prime} & \Lambda_{0} & \Lambda \\
M_{\Lambda}^{\prime} & M_{\Lambda_{0}} & M_{\Lambda}
\end{array}\right] .
\end{aligned}
$$

The many-electron ket-state

$$
\begin{aligned}
& \left|\Psi_{E} \Lambda M_{\Lambda}\right\rangle \\
& \quad \equiv\left|\left(n_{1} \lambda_{1}^{N_{1}} \alpha_{1} \Lambda_{1}, \ldots, n_{k} \lambda_{k}^{N_{k}} \alpha_{k} \Lambda_{k}\right){ }^{E} \Lambda M_{\Lambda}\right\rangle \\
& \quad={ }^{4} A_{1}
\end{aligned}
$$

and the bra-state

$$
\begin{aligned}
& \left\langle\Psi_{E^{\prime}} \Lambda M_{\Lambda}\right| \\
& \quad \equiv\left\langle\left(n_{1} \lambda_{1}^{N_{1}} \alpha_{1} \Lambda_{1}, \ldots, n_{k} \lambda_{k}^{N_{k}} \alpha_{k} \Lambda_{k}\right){ }^{E^{\prime \prime}} \Lambda M_{\Lambda}\right| \\
& \quad={ }^{4} A_{2}
\end{aligned}
$$

with $k$ shells are presented by block diagrams ${ }^{4} A_{1}$ and ${ }^{4} A_{2}$, respectively. Each free line except the last one of ${ }^{4} A_{1}$ and ${ }^{4} A_{2}$ are associated with a shell $n_{i} \lambda_{i}^{N} \alpha_{i} \Lambda_{i} M_{\Lambda_{i}}$. The remaining free lines describe the resulting momenta $\Lambda M_{\Lambda}$ of the bra- and ket-states. An arbitrary $N$-electron operator $T_{m_{\gamma}}^{(\gamma)}$ is schematically given by a block diagram ${ }^{4} A_{3}$. The $\gamma, m_{\gamma}$ line represents the rank $\gamma$ and its projection $m_{\gamma}$. The remaining lines of ${ }^{4} A_{3}$ indicate the coordinates on which the operator acts. $E, E^{\prime \prime}$, and $\Theta$ denote the coupling schemes of angular momenta of states and operators. The above-mentioned block diagrams, when specifying the data of the task considered, describe the starting positions of graphical evaluation of the $N$-electron matrix element

$$
\left\langle\Psi_{E^{\prime \prime}} \Lambda_{E}^{\prime \prime} M_{E}^{\prime \prime}\left|T_{m_{\gamma}}^{(\gamma)}\right| \Psi_{E} \Lambda_{E} M_{E}\right\rangle
$$


Due to the symmetry properties of the wave function the one-electron $F(32)$ and two-electron $G(38)$ operators in the ordinary $\mathrm{CR}$ are given in the form $[12,48]$

$$
F \doteq N f_{N}
$$

and

$$
G \doteq \frac{1}{2} N(N-1) g_{N-1 N}
$$

Here the symbol $\doteq$ indicates that the relations (63) and (64) are used only when considering the matrix elements of these operators. It is assumed that the operator $f_{N}$ acts on the $N$ th electron whereas the operator $g_{N-1 N}$ acts on the $(N-1)$ th and $N$ th electrons of the bra- and ket-states, respectively.

The common feature of all approaches of CR consists of the need to decouple the electrons (referred to as active electrons) from the wave function in order to form the matrix elements for $f_{N}$ or $g_{N-1 N}[39,42,43]$. For example, consider the case of one-electron operator $F$. Then the block diagram ${ }^{4} A_{3}$ is replaced by ${ }^{3} A_{1}$ which is connected with the matrix element $\left(n_{i} \lambda_{i} m_{i}\left|f_{m_{\gamma}}^{(\gamma)}\right| n_{j} \lambda_{j} m_{j}\right)$. More precisely, ${ }^{4} A_{3}$ is replaced by $\left({ }^{4} A_{4}[i\|f(\gamma)\| j]\left(n_{i} l_{i}|f(r)| n_{j} l_{j}\right)\right)$, where ${ }^{4} A_{4}$ represents CGC. Let us consider the position when $f_{N}$ operates on the ket-state $\left|n \lambda^{N} \alpha \Lambda M_{\Lambda}\right\rangle$. To describe this action one can use the diagram ${ }^{4} A_{5}$ obtained on the ground of Eq. (59). The rectangular $e$ is associated with $\left(\lambda^{N} \alpha \Lambda \| \lambda^{N-1}\left(\alpha^{\prime} \Lambda^{\prime}\right) \lambda\right)$. In the same way, the action of $f_{N}$ on the bra-state is considered keeping in mind that $\left\langle n \lambda^{N} \alpha \Lambda M_{\Lambda}|=| n \lambda^{N} \alpha \Lambda M_{\Lambda}\right\rangle^{+}$. After the summations over $\alpha^{\prime} \Lambda^{\prime}, m_{i}, m_{j}$ that are carried out by joining the corresponding lines of the block diagrams ${ }^{4} A_{1},{ }^{4} A_{2},{ }^{4} A_{3}$ and after the application of the Wigner-Eckart theorem, we obtain the diagram ${ }^{4} B_{1}$ which represents SBE of $F$. The rectangulars $e 1$ and $e 2$ are associated with $\left(\lambda^{N} \alpha \Lambda \| \lambda^{N-1}\left(\alpha^{\prime} \Lambda^{\prime}\right) \lambda\right)$ and $\left(\lambda^{N-1}\left(\alpha^{\prime} \Lambda^{\prime}\right) \lambda \| \lambda^{N} \alpha^{\prime \prime} \Lambda^{\prime \prime}\right)$, respectively. The symbolic expression for ${ }^{4} B_{1}$ can be written as

$$
\begin{aligned}
& {\left[\Psi_{E^{\prime \prime}} \Lambda_{E}^{\prime \prime}\left\|F^{(k)}\right\| \Psi_{E} \Lambda_{E}\right]} \\
& =\sum_{\alpha^{\prime} \Lambda^{\prime}} K\left(\lambda^{N-1}\left(\alpha^{\prime} \Lambda^{\prime}\right) \lambda \| \lambda^{N} \alpha^{\prime \prime} \Lambda^{\prime \prime}\right) \\
& \quad \times\left(\lambda^{N} \alpha \Lambda \| \lambda^{N-1}\left(\alpha^{\prime} \Lambda^{\prime}\right) \lambda\right)\left[\lambda\left\|f^{(\gamma)}\right\| \lambda\right] \\
& \quad \times\left(n_{i} l_{i}|f(r)| n_{j} l_{j}\right)^{4} B_{2} .
\end{aligned}
$$

The diagram ${ }^{4} B_{2}$ (this diagram is not presented explicitly) is obtained when the factors presented in Eq. (65) are removed from ${ }^{4} B_{1}$. In Eq. (65) the product of phase and weight factors is denoted by $K$. The phase factor is connected with antisymmetrization of the state function [41]. The weight factor describes equal contributions of different electron distributions in the state functions. Both factors are described by different algebraic formulas for $F$ and $G$ operators (see, for example, $[41,42])$.

It is easy to notice that by cutting the lines $\Lambda^{\prime \prime}, \Lambda$, and $\gamma$ (using JLV Theorems), we can decompose the diagram ${ }^{4} B_{1}$ into two separate diagrams. Suppose we consider $f^{(\gamma)}$ with $\gamma=k 0$. Then one diagram represents the RC (it is connected with $6 j$ symbol) and jointly with CFP of Eq. (65) forms the expression of SBE for the double-tensor $W^{(k 0)}[10,12]$ :

$$
\begin{aligned}
{\left[n \lambda^{N} \alpha^{\prime \prime} L^{\prime \prime} S^{\prime \prime}\left\|W^{(k 0)}\right\| n \lambda^{N} \alpha L S\right] } \\
=N \sum_{\alpha^{\prime} L^{\prime} S^{\prime}}\left(\lambda^{N-1}\left(\alpha^{\prime} L^{\prime} S^{\prime}\right) \lambda \| \lambda^{N} \alpha^{\prime \prime} \Lambda^{\prime \prime}\right) \\
\quad \times\left(\lambda^{N} \alpha L S \| \lambda^{N-1}\left(\alpha^{\prime} L^{\prime} S^{\prime}\right) \lambda\right) \\
\quad \times(-1)^{l+k+L^{\prime}+L^{\prime \prime}}[L, l]^{1 / 2}\left\{\begin{array}{ccc}
L^{\prime} & l & L \\
k & L^{\prime \prime} & l
\end{array}\right\} \delta\left(S^{\prime \prime}, S\right) .
\end{aligned}
$$

The left diagram ${ }^{4} B_{3}$ is also connected with the $\mathrm{RC}$ whose structure depends on the coupling schema $E$ and $E^{\prime \prime}$. Usually, ${ }^{4} B_{3}$ is designated as a block of spectator (inactive) electrons [42]. Finally, it is obtained that

$$
\begin{aligned}
& {\left[\Psi_{E^{\prime \prime}} \Lambda_{E}^{\prime \prime}\left\|F^{(k)}\right\| \Psi_{E} \Lambda_{E}\right]} \\
& =K\left[n \lambda^{N} \alpha^{\prime \prime} L^{\prime \prime} S^{\prime \prime}\left\|W^{(k 0)}\right\| n \lambda^{N} \alpha L S\right] \\
& \quad \times\left[\lambda\left\|f^{(\gamma)}\right\| \lambda\right]\left(n_{i} l_{i}|f(r)| n_{j} l_{j}\right)^{4} B_{3} .
\end{aligned}
$$

The diagram for the SBE when $F$ acts between shells can be easily generated following the way just described. Note that in this case the CFP has to be attributed to different shells.

The graphical evaluation for the two-electron operator $G$ has a burden of the variety of ways the operator acts on the shells of many-shell bra- and ket-states. According to ${ }^{4} A_{5}$ each of four lines of ${ }^{3} B_{1}$ generates the node in ${ }^{4} B_{1}$. The situation can be simplified (by saving the inclusion of extra nodes and sums in ${ }^{4} B_{1}$ ) when $g_{N-1 N}$ operates on the same shell of bra- and/or ket-state. For this, the relation (60) has to be employed. Then, the wave functions $\left|n \lambda^{N-2} \alpha^{\prime} \Lambda^{\prime} M_{\Lambda}^{\prime}\right\rangle$ and $\left|n \lambda^{2} \Lambda_{0} M_{\Lambda_{0}}\right\rangle$ instead of $\left|n \lambda^{N-1} \alpha^{\prime} \Lambda^{\prime} M_{\Lambda}^{\prime}\right\rangle$ and $\left|n \lambda m_{\lambda}\right\rangle$ have to be used in ${ }^{4} A_{5}$ 
and now the node of ${ }^{4} A_{5}$ is associated with a twoparticle CFP $\left(\lambda^{N} \alpha \Lambda \| \lambda^{N-2}\left(\alpha^{\prime} \Lambda^{\prime}\right) \lambda^{2}\left(\Lambda_{0}\right)\right)$. Furthermore, in the case when $G$ acts in the space of states of one shell of equivalent electrons, the structure of SBE of $G$ is similar to that of (67), where SBE of $W^{(k 0)}$ has to be replaced by SBE of the two-electron operator of the left of Eq. (50). In addition, the matrix element of $g_{\alpha \beta}$ is defined with antisymmetric two-electron functions $\left|n \lambda^{2} \Lambda_{0} M_{\Lambda_{0}}\right\rangle$.

In general, the graphical procedures of the reduction of a complex diagram for SBE in CR give the expression in terms of CFP, two-electron CFP, and $3 n j$ coefficients. Later on, from those quantities one is seeking to organize the SBEs of standard operators (of the type $\left.W^{\left(k k^{\prime}\right)}\right)$ in order to use numerical tables or computer codes.

In [37] following the algebraic approach of [38], the graphical evaluation of matrix elements was carried out by using the diagrammatic representation of generalized CFP. In this approach the electrons of the state function of $\left|\Psi_{E} \Lambda M_{\Lambda}\right\rangle$ is ordered in the way that the $n$ separated (active) electrons are considered as the latest $n$th particles of the of $N$-electron wave function. Then the generalized CFP are expressed as products of CFP and the RC. In [35] and [36] as well as in [47] the $N$-electron state function $\left|\Psi_{E} \Lambda M_{\Lambda}\right\rangle$ is generated by using the recursive relations.

Let us consider the graphical approach $[55,58,59]$ which allows the evaluation of the SBE in SQR. Below we follow the paper [59] to describe this method. In SQR a ket-state of equivalent electrons can be given by [16]

$$
\left|n \lambda^{N} \alpha \Lambda M_{\Lambda}\right\rangle=\varphi\left(n \lambda^{N} \alpha\right)_{M_{\Lambda}}^{(\Lambda)}|0\rangle={ }^{4} C_{1}|0\rangle,
$$

where $\varphi\left(n \lambda^{N} \alpha\right)_{M_{\Lambda}}^{(\Lambda)}$ is an irreducible tensorial operator of the rank $\Lambda$ composed of $N$ creation operators $a^{(\lambda)}$ and is connected with the diagram ${ }^{4} C_{1} \cdot|0\rangle$ denotes a vacuum state function. The bra-state $\left\langle n \lambda^{N} \alpha \Lambda M_{\Lambda}\right|=$ $\left|n \lambda^{N} \alpha \Lambda M_{\Lambda}\right\rangle^{+}$has the expression

$$
\begin{aligned}
& \left\langle n \lambda^{N} \alpha \Lambda M_{\Lambda}\right| \\
& \quad=\langle 0|(-1)^{N(N-1) / 2+\Lambda+M_{\Lambda}} \widetilde{\varphi}\left(n \lambda^{N} \alpha\right)_{-M_{\Lambda}}^{(\Lambda)} .
\end{aligned}
$$

In (69), $\widetilde{\varphi}\left(n \lambda^{N} \alpha\right)_{M_{\Lambda}}^{(\Lambda)}$ is an irreducible tensorial operator obtained from $\varphi\left(n \lambda^{N} \alpha\right)_{M_{\Lambda}}^{(\Lambda)}$ by replacing $a^{(\lambda)}$ with $\widetilde{a}^{(\lambda)}$ [16]. The diagram ${ }^{4} C_{2}$ is associated with the operator $(-1)^{N(N-1) / 2} \widetilde{\varphi}\left(n \lambda^{N} \alpha\right)_{M_{\Lambda}}^{(\Lambda)}$. Furthermore, the many-electron ket-state is given by [59]

$$
\left|\Psi_{E} \Lambda M_{\Lambda}\right\rangle=\Psi_{E_{M_{\Lambda}}^{(\Lambda)}}|0\rangle={ }^{4} C_{3}|0\rangle
$$

where the diagram ${ }^{4} C_{3}$ represents a tensorial product $\Psi_{E_{M_{\Lambda}}^{(\Lambda)}}$ composed of the operators $\varphi\left(n_{i} \lambda_{i}^{N_{i}} \alpha_{i}\right)^{\left(\Lambda_{i}\right)}$. Similarly, the bra-state

$$
\begin{aligned}
\left\langle\Psi_{E} \Lambda M_{\Lambda}\right| & =\langle 0|(-1)^{\sum_{i>j} N_{i} N_{j}+\Lambda+M_{\Lambda}} \widetilde{\Psi}_{E_{-M_{\Lambda}}}^{(\Lambda)} \\
& =\langle 0|(-1)^{\sum_{i>j} N_{i} N_{j} 4} C_{4}
\end{aligned}
$$

is connected with ${ }^{4} C_{4}$. According to Eqs. (28) and (68)-(71), an SBE for many-electron states has the expression

$$
\begin{aligned}
& {\left[\Lambda_{E^{\prime \prime}}\left\|T^{(\gamma)}\right\| \Lambda_{E}\right]} \\
& =(-1)^{\sum_{i>j} N_{i} N_{j}}(-1)^{\Lambda_{E}-\gamma-\Lambda_{E}^{\prime \prime}}\left[\Lambda_{E^{\prime \prime}}\right]^{-1 / 2} \\
& \quad \times\left\langle 0\left|\left[\widetilde{\Psi}_{E^{\prime \prime}}^{\left(\Lambda_{E^{\prime \prime}}\right)} \times\left[T^{(\gamma)} \times \Psi_{E}\left(\Lambda_{E}\right)\right]^{(\Lambda)}\right]^{(0)}\right| 0\right\rangle \\
& =\left\langle 0\left|(-1)^{\sum_{i>j} N_{i} N_{j} 4} C_{5}\right| 0\right\rangle .
\end{aligned}
$$

Here it is assumed that $T^{(\gamma)}$ is an $N$-electron operator given in SQR of a coupled form. The diagram ${ }^{4} C_{5}$ is associated with a reduced matrix element for manyelectron case and is obtained keeping in mind the diagram ${ }^{2} D_{1}$. We have to point out that in this section the order of the second quantization operators in an algebraic expression is associated with the order that the graphical elements have appeared in a diagram.

In order to carry out the consideration of complex RME, the standard graphs were defined in [59]. When an operator $T^{(\gamma)}$ is the electron creation operator $a^{(\lambda)}$, it follows from Eq. (72) that

$$
\begin{aligned}
{\left[n \lambda^{N} \alpha \Lambda\left\|a^{(\lambda)}\right\| n \lambda^{N-1} \alpha^{\prime} \Lambda^{\prime}\right] } & =\left\langle 0\left|{ }^{4} D_{1}\right| 0\right\rangle \\
& ={ }^{4} D_{2} .
\end{aligned}
$$

The diagram ${ }^{4} D_{1}$ describes the irreducible tensorial product of three tensors: $\widetilde{\varphi}\left(n \lambda^{N} \alpha\right)^{(\Lambda)}, T^{(\gamma)}=a^{(\lambda)}$, and $\varphi\left(n \lambda^{N-1} \alpha^{\prime}\right)^{\left(\Lambda^{\prime}\right)}$. After the "integration" in the $\mathrm{SQR}$ (graphically, as in CR, such an integration is carried out by joining the semicircles associated with $\widetilde{\varphi}\left(n \lambda^{N} \alpha\right)^{(\Lambda)}, T^{(\gamma)}$, and $\varphi\left(n \lambda^{N-1} \alpha^{\prime}\right)^{\left(\Lambda^{\prime}\right)}$ and skipping the $\langle 0|$ and $|0\rangle$ ), we obtain the diagram ${ }^{4} D_{2}$ which gives the graphical definition of the SBE. Furthermore, the relationship [14]

$$
\begin{aligned}
& {\left[n \lambda^{N} \alpha \Lambda\left\|a^{(\lambda)}\right\| n \lambda^{N-1} \alpha^{\prime} \Lambda^{\prime}\right]} \\
& \quad=(-1)^{N} \sqrt{N}\left(\lambda^{N} \alpha \Lambda \| \lambda^{N-1}\left(\alpha^{\prime} \Lambda^{\prime}\right) \lambda\right)
\end{aligned}
$$

allows the conversion of the expression obtained in SQR to the ones in CR. ${ }^{4} D_{3}$ represents SBE of the op- 

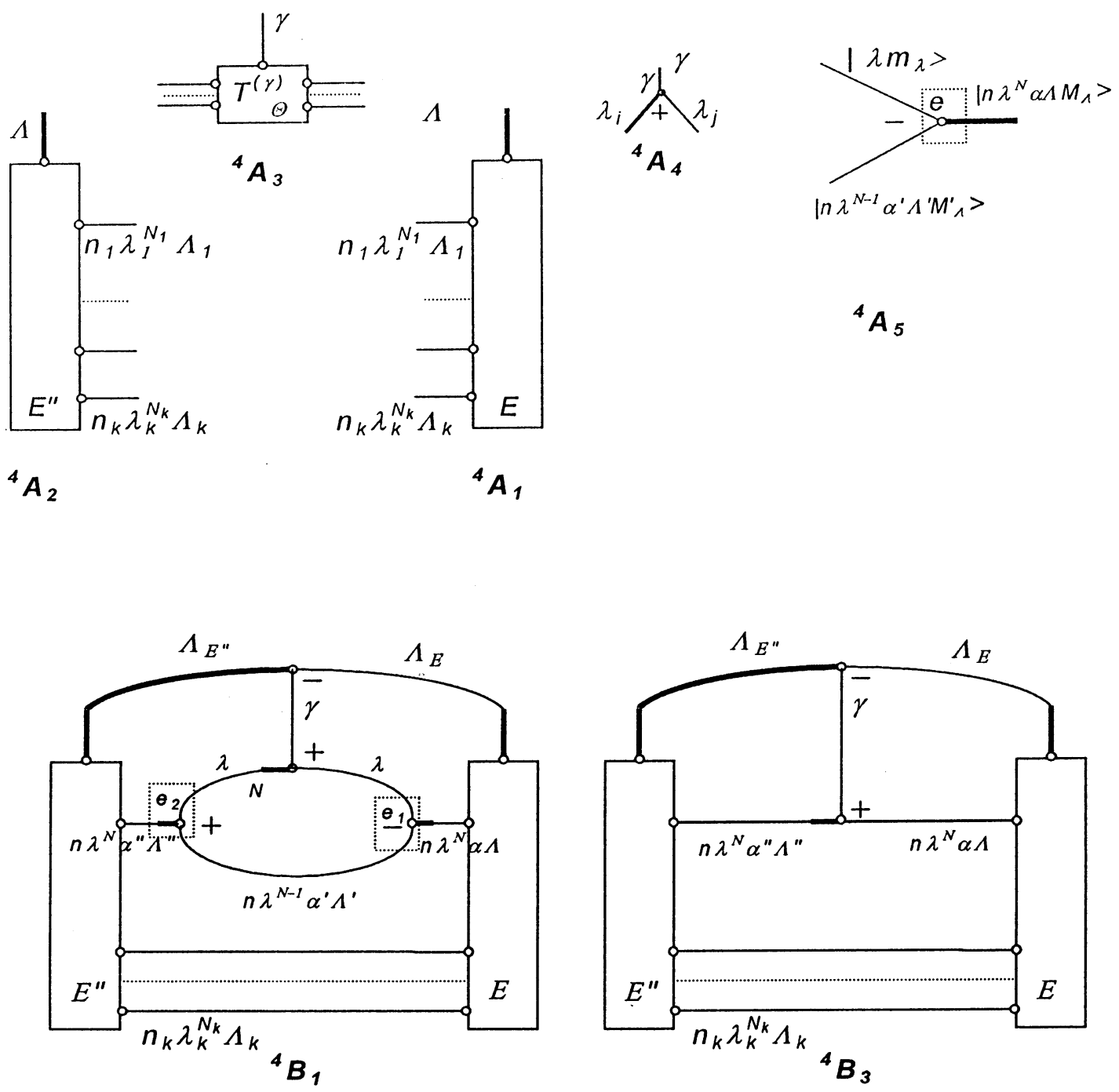

Fig. 11. Graphical evaluation of $N$-electron matrix elements in the coordinate representation.

erator $\widetilde{a}^{(\lambda)}$ which is also proportional to the CFP of Eq. (74). The diagram ${ }^{4} D_{4}$ shows SBE of the operator $W^{(\gamma)}=-\left[a^{(\lambda)} \times \widetilde{a}^{(\lambda)}\right]^{(\gamma)}$. $W^{(\gamma)}$ is a one-particle operator (40) with $\left[\lambda_{i}\left\|f^{(\gamma)}\right\| \lambda_{j}\right]=\left[\lambda_{i}\left\|w^{(\gamma)}\right\| \lambda_{j}\right]=$ $\delta\left(\lambda_{i}, \lambda_{j}\right)\left[\gamma / \lambda_{i}\right]^{1 / 2}$ (e. g., [14] or [16]). The expression of SBE of $W^{(\gamma)}$ when $\gamma=k 0$ was defined by Eq. (66). The SBE of the irreducible tensorial product of twoelectron creation operators can be written as [14]

$$
\begin{aligned}
& {\left[n \lambda^{N} \alpha \Lambda\left\|\left[a^{(\lambda)} \times a^{(\lambda)}\right]^{\left(\Lambda_{0}\right)}\right\| n \lambda^{N-2} \alpha^{\prime} \Lambda^{\prime}\right]} \\
& \quad=[N(N-1)]^{1 / 2}\left(\lambda^{N} \alpha \Lambda \| \lambda^{N-2}\left(\alpha^{\prime} \Lambda^{\prime}\right), \lambda^{2}\left(\Lambda_{0}\right)\right) .
\end{aligned}
$$

The graph of SBE for this operator is immediately obtained from ${ }^{4} D_{4}$, by replacing $\widetilde{a}^{(\lambda)}\left({ }^{2} F_{2}\right)$ with $a^{(\lambda)}\left({ }^{2} F_{1}\right)$ and the marking circle with the node CGC. According to ${ }^{2} D_{3}$, when $T^{(\gamma)}=I$ the diagram ${ }^{4} D_{5}$ is associated with $\delta\left(N \alpha \Lambda, N^{\prime} \alpha^{\prime} \Lambda^{\prime}\right)$. The projection operator onto a space of states of a shell $n \lambda^{N}$ is given by

$$
\sum_{\alpha \Lambda M_{\Lambda}}\left|n \lambda^{N} \alpha \Lambda M_{\Lambda}\right\rangle\left\langle n \lambda^{N} \alpha \Lambda M_{\Lambda}\right|=\sum_{\alpha \Lambda \Lambda^{\prime}}{ }^{4} D_{6}
$$

according to ${ }^{2} D_{6}$.

Let us study the case of many open shells. The graphical evaluation of an arbitrary matrix element of the operator $T^{(\gamma)}$ consists of decomposition of the general diagram ${ }^{4} C_{5}$ (Fig. 12) into the diagrams whose algebraic expressions are known. This procedure generates a phase factor, a RC, and SBEs. In most cases, the decomposition is performed in the way when the operators $a^{(\lambda)}$ and $\widetilde{a}^{(\lambda)}$, acting on the same shell of equivalent electrons, are placed side-by-side. Of course, the ordering just discussed is not obligatory for the method developed. The phase factor arises due to the change of the ordering of creation and annihilation 


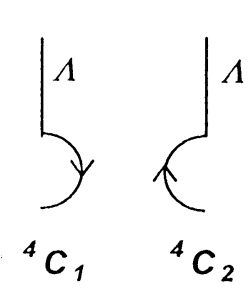

$<0 \mid$

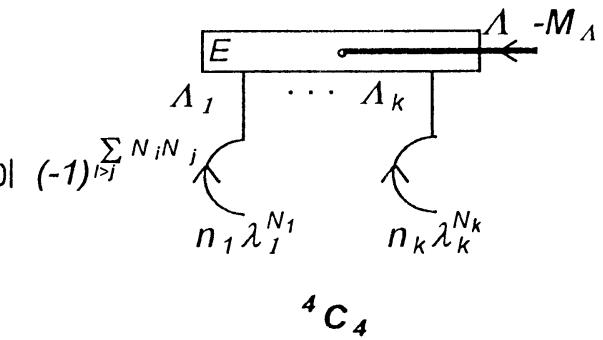

$\Lambda M_{\Lambda}$

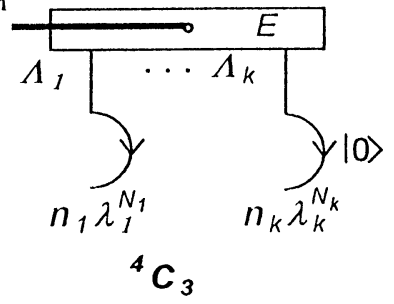

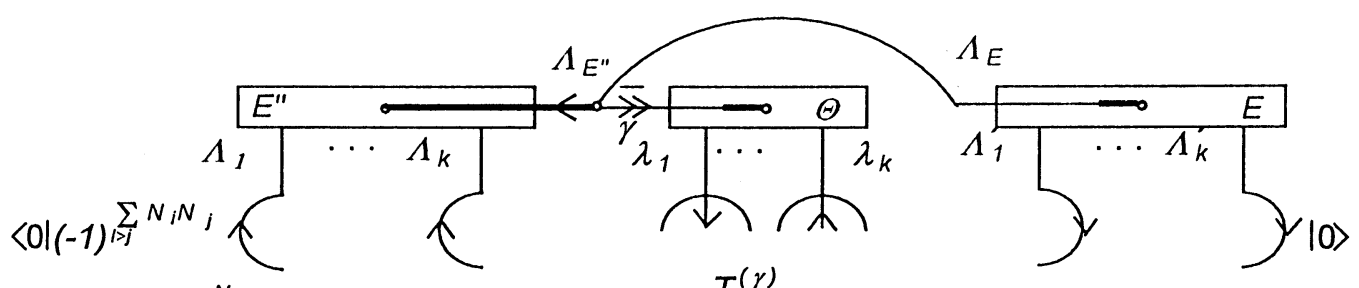

$$
n_{1} \lambda_{1}^{N_{1}} \quad n_{k} \lambda_{k}^{N_{k}}
$$

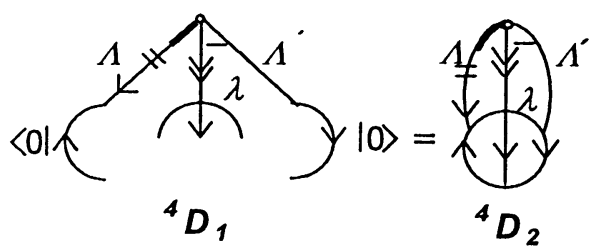

${ }^{4} C_{5}$

$$
n_{1} \lambda_{1}^{N_{i}} \quad n_{k} \lambda_{k}^{N_{k}}
$$

$<0 \mid$

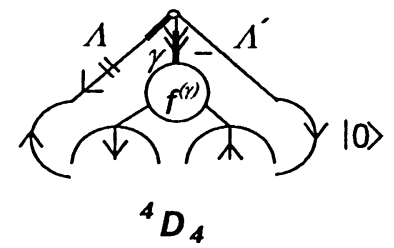

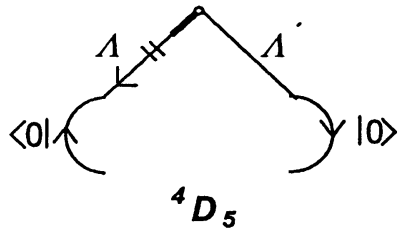

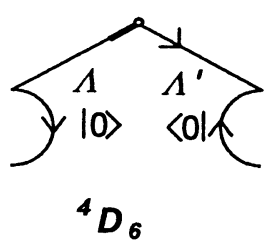

a)

$\langle 0|(-1)^{\sum_{i=1} N_{i} N}$

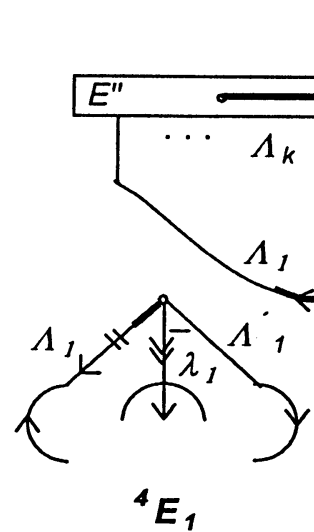

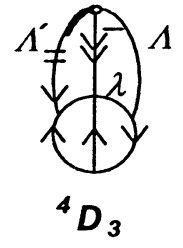

$D_{3}$

$$
\text { (1) }
$$


operators in ${ }^{4} C_{5}$ in order to form graphical elements (diagrams) which represent SBE. When changing the ordering, the crossings of the lines corresponding to the second quantization operators which are placed sideby-side occur. Then the phase factor is determined by simple formulae $(-1)^{\sum_{p} a_{p} b_{p}}$. Here $a$ and $b$ denote the number of creation and annihilation operators associated with the crossing lines. The sum over $p$ is carried out over all possible crossings (more details can be found in [59]).

Now we shall consider the two typical Situations (a) and (b) which can appear in the construction of a RC and SBE (see (a) of Fig. 12).

Situation (a). Suppose that one of the operators $a^{(\lambda)}$ and $\widetilde{a}^{(\lambda)}$ in $T^{(\gamma)}$ acts on the shell $n_{1} \lambda_{1}^{N_{1}}$. Then we transform the diagram ${ }^{4} C_{5}$ in the way that the operators $\widetilde{\varphi}\left(n_{1} \lambda_{1}^{N_{1}} \alpha_{1}\right)^{\left(\Lambda_{1}\right)}, a^{\left(\lambda_{1}\right)}$, and $\varphi\left(n_{1} \lambda_{1}^{N_{1}^{\prime}} \alpha_{1}^{\prime}\right)^{\left(\Lambda_{1}^{\prime}\right)}$ have to be placed side-by-side. Later on, by using JLV Theorems (we cut the lines $\Lambda_{1}, \lambda_{1}$, and $\Lambda_{1}^{\prime}$ ) we decompose the diagram ${ }^{4} C_{5}$ and obtain ${ }^{4} E_{1}$ which is associated with $\left[n_{1} \lambda_{1}^{N_{1}} \alpha_{1} \Lambda_{1}\left\|a^{\left(\lambda_{1}\right)}\right\| n_{1} \lambda_{1}^{N^{\prime}} \alpha_{1}^{\prime} \Lambda_{1}^{\prime}\right]$.

Situation (b). Suppose that the operator $T^{(\gamma)}$ does not act on some shell of equivalent electrons, say, $n_{k} \lambda_{k}$. Then we place the operators $\widetilde{\varphi}\left(n_{k} \lambda_{k}^{N_{k}} \alpha_{k}\right)^{\left(\Lambda_{k}\right)}$ and $\varphi\left(n_{k} \lambda_{k}^{N_{k}^{\prime}} \alpha_{k}^{\prime}\right)^{\left(\Lambda_{k}^{\prime}\right)}$ side-by-side and cut the lines $\Lambda_{k}$ and $\Lambda_{k}^{\prime}$ to form ${ }^{4} E_{2}$ (see also ${ }^{2} D_{3}$ and ${ }^{4} D_{5}$ ) which is associated with $\delta\left(N_{k} \alpha_{k} \Lambda_{k}, N_{k}^{\prime} \alpha_{k}^{\prime} \Lambda_{k}^{\prime}\right)$. In this way we examine the operator $T^{(\gamma)}$ and all shells in the bra- and ket-functions. At the end of this procedure, we obtain the graphical expression for SBE in terms of the product of RC ${ }^{4} E_{2}$ and SBEs of the types ${ }^{4} E_{1}$ and ${ }^{4} E_{3}$.

Let us return now to the case studied in the $\mathrm{CR}$ at the beginning of this section, i. e. when $T^{(\gamma)}$ represents a one-particle operator $F$ which operates only on one shell (say, $n_{1} \lambda_{1}^{N_{1}}$ ) of equivalent electrons. In SQR the expression for the RME is immediately obtained referring to procedures of Situations (a) and (b) and the graphs ${ }^{3} A_{3}$ and ${ }^{4} D_{4}$ :

$$
\begin{aligned}
& {\left[\Psi_{E^{\prime \prime}} \Lambda_{E}^{\prime \prime}\left\|F^{(k)}\right\| \Psi_{E} \Lambda_{E}\right]} \\
& =(-1)^{\varphi_{a}}\left[n_{1} \lambda_{1}^{N_{1}} \alpha_{1} \Lambda_{1}\left\|W^{(\gamma)}\right\| n_{1} \lambda_{1}^{N_{1}} \alpha_{1}^{\prime} \Lambda_{1}^{\prime}\right] \\
& \quad \times M C\left(\lambda_{1}, \lambda_{1}, f^{(\gamma)}\right)^{4} E_{2} .
\end{aligned}
$$

The phase factor $(-1)^{\varphi_{a}}$ can be determined by the rule described above. When $\gamma=k 0$ the formula (77) agrees with (67). The diagram ${ }^{4} E_{2}$ corresponds to ${ }^{4} B_{3}$.

For further illustration of the use of the technique developed, consider the operator $\left[a^{\left(\lambda_{1}\right)} \times \widetilde{a}^{\left(\lambda_{2}\right)}\right]^{(\gamma)}$. Ac- tually, this is the case when $F$ acts on the states of two shells. Assume also that $\left|\Psi_{E} \Lambda M_{\Lambda}\right\rangle$ is formed from two open shells. The diagram ${ }^{4} C_{5}$ for this problem corresponds to ${ }^{2} E_{1}$. Then the expression for RME can be found by directly applying the formulae (23) or (29) when $j=\Lambda, j^{\prime}=\Lambda^{\prime}, \gamma=k$ and $j_{i}=\Lambda_{i}, j_{i}^{\prime}=\Lambda_{i}^{\prime}$, $k_{i}=\lambda_{i}(i=1,2)$ :

$$
\begin{aligned}
& {\left[n_{1} \lambda_{1}^{N_{1}} \alpha_{1} \Lambda_{1} n_{2} \lambda_{2}^{N_{2}} \alpha_{2} \Lambda_{2} \Lambda \|\left[a^{\left(\lambda_{1}\right)} \times \widetilde{a}^{\left(\lambda_{2}\right)}\right]^{(\gamma)}\right.} \\
& \left.\quad \times \| n_{1} \lambda_{1}^{N_{1}^{\prime}} \alpha_{1}^{\prime} \Lambda_{1}^{\prime} n_{2} \lambda_{2}^{N_{2}^{\prime}} \alpha_{2}^{\prime} \Lambda_{2}^{\prime} \Lambda^{\prime}\right] \\
& =(-1)^{N_{1}-1} \bar{C}_{4}^{4} D_{2}{ }^{4} D_{3} .
\end{aligned}
$$

So, in order to obtain the SBE for the many-electron case, we have simply to replace the $\operatorname{SBE}^{2} A_{4}\left(A^{\left(k_{1}\right)}\right)$ and ${ }^{2} A_{4}\left(B^{\left(k_{2}\right)}\right)$ in (23) with the SBE of $a^{\left(\lambda_{1}\right)}\left({ }^{4} D_{2}\right)$ and $\widetilde{a}^{\left(\lambda_{2}\right)}\left({ }^{4} D_{3}\right)$, respectively. The phase factor $(-1)^{N_{1}-1}$ is obtained keeping in mind the phase factor $(-1)^{N_{1} N_{2}}$ from Eq. (72) and the phase factor $(-1)^{\sum_{p} a_{p} b_{p}}$ due to three crossings of the lines $j_{1}^{\prime}, k_{1}, j_{2}, k_{2}$ (see the diagram ${ }^{2} E_{2}$ ).

To illustrate the derivation of expressions for a twoparticle operator, consider the diagram ${ }^{3} D_{1}$ (53). When this operator acts on one shell of equivalent electrons, we have to determine the expression for SBE of ${ }^{3} D_{2}$. Such an expression can be easily given in terms of CFP by using Eqs. (74) and (76). As discussed in Section 3 , the case when ${ }^{3} D_{1}$ operated on two shells gave the scalar operator $\left[W^{(x)}\left(n_{1} \lambda_{1}\right) \times W^{(x)}\left(n_{2} \lambda_{2}\right)\right]^{(0)}$ as a result. The expression for SBE of this operator is obtained from Eq. (78) by replacing $a^{\left(\lambda_{1}\right)}$ and $\widetilde{a}^{\left(\lambda_{2}\right)}$ with $W^{(x)}\left(n_{1} \lambda_{1}\right)$ and $W^{(x)}\left(n_{2} \lambda_{2}\right)$, respectively. Then the phase factor in (78) is equal to one. The detailed study of SBE for a two-particle operator $G$ in the approach discussed can be found in $[59,64]$.

Generalizing the discussion of evaluation of SBE in SQR one could conclude that the important advantage of the method considered is that all calculations are carried out in SQR, and therefore, antisymmetric conditions are ensured automatically. It is also significant that in the SQR approach unlike to that of CR first of all the operator with suitable tensorial structure and ordering of $a^{(\lambda)}$ and $\widetilde{a}^{(\lambda)}$ is formed by putting the operators of the second quantization side-by-side and only later the expression for SBE is derived if necessary. In addition, we express SBE in terms of the irreducible tensorial product of the second quantization operators, and therefore, the angular momentum and antisymmetric features of the quantity studied are comprised by the same graph. In CR, CFP is introduced into the graphi- 
cal evaluations in an artificial way by symbolically denoting it as a square or rectangular $[41,42,48]$ on the nodes of angular momentum diagrams.

As mentioned in the introduction, the solution of many recent atomic spectroscopy problems required very extensive calculations and the graphical methods could be used for improving the algorithm for computer codes. Already in the middle of the sixties in [73-75] the computers have been used to generate $3 n j$ coefficients, to perform their classification as well as decomposition. Recently there are a number of computer codes which derive expressions for recoupling coefficients by using the JLV Theorems (see e. g., [76-81]). The programs $[82,83]$ which use the graphical methods described in the present section to derive the expressions for matrix elements can be singled out. The computer code [84] to calculate the energy spectra of open-shell atoms by using MBPT was coded by using the graphical method in SQR described in Sections 4 and 5.

\section{Concluding remarks}

The graphical method of angular momentum theory proposed by A. Jucys and co-workers is an efficient and widely used tool in the theoretical spectroscopy investigations. The pioneering studies in the developing of the principles of the method as well as pioneering works in the application of the method in perturbation theory, graphical evaluation of matrix elements of the operators stimulated numerous researchers for further developments of the graphical methods and techniques.

The graphical method developed in the second quantization representation is supplied by new features in comparison to the method elaborated in the coordinate representation. In the second quantization approach, the introduced diagrams for the operators, wave functions, and matrix elements not only describe the momentum properties of these quantities, but also the antisymmetry conditions are ensured automatically because the creation and annihilation operators are included into diagrams explicitly. The submatrix element is associated with the diagram representing the scalar tensorial product of three tensors (expressed in the second quantization representation) linked with $N$-electron bra- and ket-wavefunctions and the operator under investigation. The method developed is very efficient and flexible while treating many-electron open-shell atoms.

Many researchers associate Jucys graphs (or Jucys, Levinson, and Vanagas graphs) associate with the di- agrams constructed from the Wigner coefficients (see Section 1). However, as it was pointed out in the present paper, Prof. A. Jucys and his co-workers have also proposed the graphs for the irreducible tensors and their submatrix elements as well as introduced the effective graphical method based on the Clebsch-Gordan coefficients. Later on the basis of this method the graphical approach in the second quantization representation was developed. Therefore, the author of the paper would like to suggest to expend the concept of "Jucys graphs" by accumulating into it the diagrams for the operators and submatrix elements generated in the second quantization representation (Sections 4 and 5).

\section{References}

[1] A.P. Jucys, J. Levinson, and V.V. Vanagas, Judejimo kiekio momento teorijos matematinis aparatas (Lietuvos TSR valstybinès politinès ir mokslinès literatūros leidykla, Vilnius, 1960) [in Russian]; Mathematical Apparatus of the Theory of Angular Momentum (Israel Program for Scientific Translation, Jerusalem, 1962) [English translation]; Mathematical Apparatus of the Theory of Angular Momentum (Gordon and Breach, New York, 1964) [English translation].

[2] E.U. Condon and G.H. Shortley, Theory of Atomic Spectra (Cambridge University Press, New York, 1935).

[3] E. Wigner, Gruppentheorie und ihre Anwendung auf die Quantenmechanik der Atomspektren (Braunschweig, 1931).

[4] E.P. Wigner, Group Theory and Its Application to the Quantum Mechanics of Atomic Spectra (Academic Press, New York, 1959).

[5] G. Racah, Phys. Rev. 61, 186 (1941).

[6] G. Racah, Phys. Rev. 62, 438 (1942).

[7] G. Racah, Phys. Rev. 63, 367 (1943).

[8] G. Racah, Phys. Rev. 76, 1352 (1949).

[9] A.R. Edmonds, Angular Momentum in Quantum Mechanics (Princeton University Press, Princeton, 1960).

[10] B.R. Judd, Operator Techniques in Atomic Spectroscopy (McGraw-Hill, New York, 1963).

[11] U. Fano and G. Racah, Irreducible Tensorial Sets (Academic Press, New York, 1959).

[12] A.P. Jucys and A.J. Savukynas, Mathematical Foundations of the Atomic Theory (Mokslas, Vilnius, 1973).

[13] A.P. Jucys, Selected Papers (Mokslas, Vilnius, 1978) [in Russian].

[14] B.R. Judd, Second Quantization in Atomic Spectroscopy (Johns Hopkins, Baltimore, 1967).

[15] L. Armstrong, Jr., Phys. Rev. 172, 18 (1968).

[16] Z. Rudzikas and J. Kaniauskas, Quasispin and Isospin in the Theory of Atom (Mokslas, Vilnius, 1984). 
[17] Z. Rudzikas, Theoretical Atomic Spectroscopy (Cambridge University Press, Cambridge, 1997).

[18] I.B. Levinson, FTI Darbai 2, 17 (1956) [in Russian].

[19] I.B. Levinson, FTI Darbai 2, 31 (1956) [in Russian].

[20] I.B. Levinson, Lietuvos MA Darbai, Ser. B 4, 3 (1957) [in Russian].

[21] I.B. Levinson and V.V. Vanagas, Opt. Spektrosk. (USSR) 2, 3 (1957).

[22] V.V. Vanagas and J. Čiplys, Lietuvos MA Darbai, Ser. B 3, 15 (1958) [in Russian].

[23] A.M. Gutman and S. Budryte, Lietuvos MA Darbai, Ser. B 4, 3 (1959) [in Russian].

[24] J. Čiplys, S. Budryte, and A.P. Jucys, Liet. Fiz. Rink. 1(3-4), 263 (1961).

[25] S. Budrytė and A.P. Jucys, Liet. Fiz. Rink. 1, 281 (1961).

[26] A. Bandzaitis, J. Vizbaraite, and A. Jucys, Liet. Fiz. Rink. 2, 61 (1962).

[27] A. Bandzaitis, J. Vizbaraite, and A. Jucys, Liet. Fiz. Rink. 2, 73 (1962).

[28] A. Bandzaitis, J. Vizbaraite, and A. Jucys, Liet. Fiz. Rink. 2, 91 (1962).

[29] A. Bandzaitis, J. Vizbaraite, and A. Jucys, Liet. Fiz. Rink. 2, 109 (1962).

[30] A.P. Jucys and A.A. Bandzaitis, Theory of Angular Momentum in Quantum Mechanics, 1st edn. (Mokslas, Vilnius, 1965).

[31] A.P. Jucys and A.A. Bandzaitis, Theory of Angular Momentum in Quantum Mechanics, 2nd edn. (Mokslas, Vilnius, 1977).

[32] A.P. Jucys, Z.B. Rudzikas, and A.A. Bandzaitis, Liet. Fiz. Rink. 5, 1 (1965).

[33] A. Bolotin, I. Levinson, and V. Tolmachev, Liet. Fiz. Rink. 4, 25 (1964).

[34] J. Goldstone, Proc. Roy. Soc. London Ser. A 239, 267 (1957).

[35] A. Matulis, E. Našlènas, and A. Bandzaitis, Liet. Fiz. Rink. 5, 453 (1965).

[36] A. Matulis and A. Bandzaitis, Liet. Fiz. Rink. 5, 289 (1965).

[37] G. Kamuntavičius, Liet. Fiz. Rink. 7, 553 (1967).

[38] I.B. Levinson, Lietuvos MA Darbai, Ser. B 4, 17 (1957) [in Russian].

[39] V. Tutlys, Automatization of calculations of the atomic theory by using the nonorthogonal orbitals, Thesis (Vilnius, 1972).

[40] Z.I. Kupljauskis, A.V. Kupljauskiene, and V. Tutlis, Izv. Vuzov Ser. Fiz. (3), 7-11 (1981) [Sov. Phys. Tr. (3), 203 (1981)].

[41] J.S. Briggs, Rev. Mod. Phys. 41, 189 (1971).

[42] K.-N. Huang, Rev. Mod. Phys. 51, 216 (1979).

[43] D.M. Brink and G.R. Satchler, Angular Momentum, 2nd edn. (Clarendon Press, Oxford, 1968).

[44] E. El Bas and B. Castel, Graphical Methods of Spin Algebras in Atomic, Nuclear, and Particle Physics (Marcel Dekker, New York, 1972).
[45] P.G.H. Sandars, La structure hyperfine magnetique des atoms et des molecules (Editions du Centre National de la Récherche Scientifique, Paris, 1967).

[46] P.G.H. Sandars, Adv. Chem. Phys. 14, 365 (1969).

[47] V. Tolmachev, Adv. Chem. Phys. 14, 421 (1969).

[48] I. Lindgren and M. Morrison, Atomic Many-Body Theory, Springer Series in Chemical Physics, Vol. 13, 2nd edn. (Springer-Verlag, Berlin, 1982).

[49] D.A. Varshalovich, A.N. Moskalev, and V.K. Khersonskii, Quantum Theory of Angular Momentum (World Scientific, Singapore, 1988).

[50] G. Merkelis, J. Kaniauskas, and Z. Rudzikas, Liet. Fiz. Rink. 25, 21 (1985).

[51] G. Merkelis and G. Gaigalas, Irreducible tensorial form of effective operator in the perturbation theory of open-shell atoms, in: Abstracts of the Conference "Theory of Atoms and Atomic Spectra" (Minsk, Byelorussia, 1983).

[52] G. Merkelis, G. Gaigalas, and Z. Rudzikas, Liet. Fiz. Rink. 25, 14 (1985).

[53] G. Gaigalas, J. Kaniauskas, and Z. Rudzikas, Liet. Fiz. Rink. 25, 3 (1985).

[54] G. Merkelis, G. Gaigalas, J. Kaniauskas, and Z. Rudzikas, Izv. Vuzov Ser. Fiz. 50, 1403 (1986).

[55] G. Gaigalas and G. Merkelis, Acta Phys. Hung. 61, 111 (1987).

[56] G. Merkelis and G. Gaigalas, The graphical method of construction and the calculation of spin-angular parts of operator's expansion for many-body perturbation theory for atoms with several open shells, in: Energy Levels and Transition Probabilities in Atoms and Ions (Scientific Council of Spectroscopy, Moscow, 1985) p. 20 .

[57] G. Merkelis, Physica Scripta 61, 662 (2000).

[58] G. Merkelis, Lithuanian J. Phys. 38, 247 (1998).

[59] G. Merkelis, Physica Scripta 63, 289 (2001).

[60] G. Merkelis, Lithuanian J. Phys. 41, 63 (2001).

[61] G. Merkelis and R. Karazija, J. Electron Spectr. Relat. Phenom. 133, 123 (2003).

[62] G. Gaigalas and Z. Rudzikas, J. Phys. B29, 3303 (1996).

[63] G. Gaigalas, Z. Rudzikas, and Ch. Froese Fischer, J. Phys. B 30, 3747 (1996).

[64] G. Gaigalas, Lithuanian J. Phys. 39, 79 (1998).

[65] I.P. Grant, in: Methods in Computational Chemistry, Vol. 2, ed. S. Wilson (Plenum Press, New York, 1988) p. 1.

[66] P.H.M. Uylings, J. Phys. B 25, 4391 (1992).

[67] R. Karazija, Sums of Atomic Quantities and Mean Characteristics of Spectra (Mokslas, Vilnius, 1991).

[68] L. Rudzikaitè and R. Karazija, Liet. Fiz. Rink. 29, 143 (1989).

[69] A.P. Jucys and J. Vizbaraite, Lietuvos MA Darbai, Ser. B 4(27), 45 (1961). 
[70] U. Fano, Phys. Rev. 140, A67 (1965).

[71] V. Tutlys and A. Kupliauskiene, Lithuanian J. Phys. 41, 31 (2001).

[72] A. Kupliauskienė, N. Rakštikas, and V. Tutlys, J. Phys. B 34, 1783 (2001).

[73] P. Rumšas, V. Matulis, and A. Jucys, Liet. Fiz. Rink. 4, 447 (1964).

[74] P. Rumšas, A. Bandzaitis, and A. Jucys, Liet. Fiz. Rink. 2, 197 (1965).

[75] A. Bandzaitis and D. Stulpinas, Liet. Fiz. Rink. 7, 27 (1967).

[76] P.G. Burke, Comput. Phys. Commun. 1, 241 (1970).

[77] A. Bar-Shalom and M. Klapisch, Comput. Phys. Commun. 50, 375 (1988).

[78] P.M. Lima, Comput. Phys. Commun. 66, 89 (1991).
[79] V. Fack, S.N. Piter, and J. Van der Jeugt, Comput. Phys. Commun. 101, 155 (1997).

[80] S. Fritzsche, T. Inghoff, T. Bastug, and M. Tomaselli, Comput. Phys. Commun. 39, 314 (2001).

[81] D. Van Dyck and V. Fack, Comput. Phys. Commun. 154, 219 (2003).

[82] V. Tutlys, A program to calculate the matrix elements in multiconfigurational approximation, in: Collection of Programs for Mathematical Methods of Atomic Calculations, Vol. 4 (Vilnius, 1980) [in Russian].

[83] P.M. Lima, Comput. Phys. Commun. 66, 99 (1991).

[84] G. Gaigalas, M.J. Vilkas, A. Bernotas, and G. Merkelis, Program to calculate energy spectra of open-shell atoms by using MBPT, in: Abstracts of EGAS-23, eds. S. Legovski and Z. Rudzikas (Torun, Poland, 1991).

\title{
JUDĖJIMO KIEKIO MOMENTO TEORIJOS JUCIO DIAGRAMOS
}

\author{
G. Merkelis
}

VU Teorines fizikos ir astronomijos institutas, Vilnius, Lietuva

\section{Santrauka}

Darbas skirtas prof. A. Jucio 100-osioms gimimo metinèms paminèti ir apžvelgia judejjimo kiekio momento teorijos grafinio metodo pagrindiniu principu, plètros bei taikymo darbų, atliktų Vilniuje, rezultatus. Supažindinama su pagrindiniais A. Jucio, J. Levinsono ir V. Vanago grafinio metodo elementais, teoremomis bei taisyklemis. Aptariami A. Jucio ir A. Bandzaičio išplètoto grafinio metodo privalumai ir trūkumai. Nurodomos kitu autorių pasiūlytos šių metodų atmainos, igalinančios išplèsti grafiškai vaiz- duojamu dydžių ir veiksmų ratą. Žymi darbo dalis skirta grafinei technikai antrinio kvantavimo metodu aprašyti. Ji remiasi A. Jucio ir A. Bandzaičio grafiniu metodu. Jos diagramos atspindi tiek judèjimo kiekio momento teorijos savybes, tiek ir fermionų dalelių sistemos antisimetriškumo savybes, todèl tai ypač efektyvus būdas teoriškai tirti îvairius daugiaelektronių atomų ir jonų su neužpildytais sluoksniais dydžius. Aptariami grafiniai būdai atomo sąveikas nusakančių operatorių $N$-elektronių matricinių elementų išraiškoms koordinatinio ir antrinio kvantavimo vaizdavimais gauti. 